\title{
Analysis of flame curvature evolution in a turbulent premixed bluff body burner
}

Cite as: Phys. Fluids 30, 095101 (2018); https://doi.org/10.1063/1.5044525

Submitted: 13 June 2018 . Accepted: 17 August 2018 . Published Online: 05 September 2018

Luis Cifuentes (D), Cesar Dopazo (D), Anurag Sandeep, Nilanjan Chakraborty, and Andreas Kempf

\section{ARTICLES YOU MAY BE INTERESTED IN}

Statistics of strain rates and surface density function in a flame-resolved high-fidelity simulation of a turbulent premixed bluff body burner

Physics of Fluids 30, 065101 (2018); https://doi.org/10.1063/1.5029931

Application of a modified Prandtl mixing length model to the turbulent far wake with a variable mainstream flow

Physics of Fluids 30, 095102 (2018); https://doi.org/10.1063/1.5045853

Multiscale analysis of head-on quenching premixed turbulent flames Physics of Fluids 30, 105102 (2018); https://doi.org/10.1063/1.5047061 


\title{
Analysis of flame curvature evolution in a turbulent premixed bluff body burner
}

\author{
Luis Cifuentes, ${ }^{1, a)}$ Cesar Dopazo, ${ }^{2}$ Anurag Sandeep, ${ }^{1}$ Nilanjan Chakraborty, ${ }^{3}$ \\ and Andreas Kempf ${ }^{1,4}$ \\ ${ }^{1}$ Chair of Fluid Dynamics, Institute for Combustion and Gasdynamics (IVG), University of Duisburg-Essen, \\ Duisburg 47057, Germany \\ ${ }^{2}$ School of Engineering and Architecture_Fluid Mechanics Area, University of Zaragoza, C/Maria de Luna 3, \\ Zaragoza 50018, Spain \\ ${ }^{3}$ School of Mechanical and Systems Engineering, Newcastle University, Claremont Road, \\ Newcastle-Upon-Tyne NE1 7RU, United Kingdom \\ ${ }^{4}$ Center for Nano-Integration Duisburg-Essen (CENIDE), University of Duisburg-Essen, \\ Duisburg 47057, Germany
}

(Received 13 June 2018; accepted 17 August 2018; published online 5 September 2018)

\begin{abstract}
The physical mechanisms responsible for flame curvature evolution of a methane-air premixed flame attached to a bluff-body burner have been investigated using a high-fidelity flame-resolved threedimensional simulation database. The contributions to the mean curvature generation due to the fluid flow motion and due to a combination of flow and flame propagation induced strain rates have been analyzed in detail and dominant contributions in different zones (reactants, flame, and products) of the flame have been identified. The effect of fluid flow on the mean curvature evolution is important on the unburned gas side, whereas the flame propagation dominates the mean curvature evolution in the reaction region and toward the hot products. The statistical contributions of the mean curvature transport equation have been analyzed in terms of the iso-scalar surface geometry, characterized by the mean and Gauss curvatures. This information has subsequently been used to provide physical insights into the dominant mechanisms of curvature evolution for different flame topologies. Published by AIP Publishing. https://doi.org/10.1063/1.5044525
\end{abstract}

\section{INTRODUCTION}

Turbulent premixed flames are inherently curved, supposedly because of flame wrinkling induced by turbulent fluid motion. ${ }^{1}$ The curvature of premixed flames plays a key role in analyzing how turbulence affects the local flame propagation rate, ${ }^{1-31}$ the flame strain rate, ${ }^{6,9,16-20}$ the evolution of the reactive scalar gradient, and the evolution of the flame area through flame stretch. ${ }^{18,23,32-38}$ Markstein $^{2}$ introduced a phenomenological model for the burning velocity as a function of the flame front curvature. Clavin and Joulin ${ }^{3}$ examined the stability of highly wrinkled premixed flames. Activation energy asymptotics were used to examine concave and convex premixed flames with a radius of curvature comparable to the thickness of the preheat zone, ${ }^{4,5}$ aiming to analyze the statistical behaviour of flame stretch and its influence on flame propagation. Based on two-dimensional ${ }^{6,8,10-15,23}$ and three-dimensional ${ }^{7,16-20,22,24,26,27,29}$ Direct Numerical Simulation (DNS), it has been shown that local flame curvature significantly affects the statistical behaviours of displacement speed $S_{d}=|\nabla c|^{-1}(D c / D t)$ (where $c$ is the reaction progress variable) and its components for both statistically planar ${ }^{10-18,22-24,26,27,31}$ and statistically spherical flames. ${ }^{19,20,29,36}$ The curvature dependence of displacement speed has also been reported by experimental analyses. ${ }^{9,21,25,28,30}$ Consumption speed $S_{c}=-\rho_{0}^{-1} \int \dot{\omega} \mathbf{n} \cdot d \mathbf{x}$ (where $\dot{\omega}$ is the reaction progress

a)Electronic mail: luis.cifuentes@uni-due.de variable, $\mathbf{n}$ is the flame normal vector, and $\rho_{0}$ is the unburned gas density) ${ }^{6,7,21,31}$ is also affected by local flame curvature in premixed turbulent combustion. The correlation between the flame speed and curvature weakens with increasing turbulence intensity. ${ }^{12,13,18,21,26,27,31}$ These curvature dependences of flame speeds are affected by the effective Lewis number and turbulence intensity so that the flame surface temperature becomes a strong function of local flame curvature in the presence of differential diffusion of heat and mass. ${ }^{6,7,17,24,36}$ The molecular diffusion rate contribution to displacement speed can be split into flame normal and tangential components, with the latter being directly proportional to the negative mean curvature. ${ }^{6-8,10-20,22-24,26,27}$ The normal diffusion component of displacement speed also exhibits complex curvature dependence especially for non-unity Lewis number flames and flames with a non-zero global mean curvature. ${ }^{16-20}$

The correlation between consumption speed and local flame curvature has been found to be positive (negative) for characteristic Lewis numbers greater (smaller) than unity, ${ }^{7}$ and a recent DNS analysis of premixed dodecane/air flames with detailed chemical kinetics shows that the negative correlation between consumption speed and curvature decreases with increasing Karlovitz number $\mathrm{Ka}^{31}$ The curvature dependences of displacement speed measurements in a low swirl burner for several $\mathrm{H}_{2} / \mathrm{CO}$ mixtures at various flow velocities have shown that the leading edge curvature statistics are a strong function of the turbulence intensity of the flow but display a weak dependence on fuel composition. ${ }^{30}$ 
In addition to propagation, both dilatation and flame tangential strain rates in premixed flames have been found to be negatively correlated with local curvature, ${ }^{6,9,16-23,26,27}$ but the curvature dependence weakens with increasing turbulence intensity. ${ }^{12,13,18,21,26,27}$ The curvature dependences of displacement speed and tangential strain rate lead to a non-linear curvature dependence of stretch rate (which includes the tangential strain rates due to the flow and that induced by curvature). ${ }^{32,33}$ Data from DNS with simple ${ }^{15}$ and detailed chemistry ${ }^{26}$ have shown that the average stretch due to curvature can play an important role; while most flame area displays a radius of curvature greater than the laminar flame thickness, most stretch occurs in flame elements with high curvature magnitudes. The effects of flame stretch on the evolution of the flame surface area and the scalar gradient magnitude SDF have been elucidated by Pope ${ }^{32}$ and Candel and Poinsot ${ }^{33}$ (SDF stands for surface density function and quantifies the local elementary surface/volume ratio ${ }^{18,34-37}$ ). Pope $^{32}$ described the local geometry of fluid and scalar iso-surfaces by evolution equations in parametric coordinates for the position vector, unit vector normal to the surface, principal curvatures, and area stretch factor and derived a transport equation for the SDF. Candel and Poinsot ${ }^{33}$ derived the mathematical expression for flame stretch and evolution equations of flame area, volume, and surface to volume ratio. Girimaji and Pope ${ }^{38}$ used DNS datasets of isotropic turbulent velocity fields to examine the evolution of surfaces that propagate at a constant velocity and computed statistical data of tangential strain rate, fluid velocity, characteristic curvature, and area of propagating surface elements. It has been demonstrated that the curvature dependences of stretch rate, dilatation rate, and tangential strain rate give rise to complex correlations between the terms of the SDF transport equation and curvature. ${ }^{18,23,34-36}$ These correlations are also affected by the characteristic Lewis number, ${ }^{35}$ choice of the fuel-air mixture, and global mean curvature ${ }^{36}$ Moreover, the effects of curvature on the scalar dissipation rate (SDR) evolution ${ }^{39}$ are analogous to those on SDF transport. These curvature dependences of flame displacement speed and SDF have been found to be of crucial importance in the closures of Flame Surface Density (FSD) and SDR, especially in the context of Large Eddy Simulations (LES) ${ }^{40-42}$ In this respect, it is worth mentioning that probability density function (PDF) models of scalar mixing require the expected values of the molecular fluxes conditional upon the scalar value ${ }^{12}$ and the micro-mixing term must be modeled; the curvature-related diffusion must be described in terms of calculable statistical moments or, alternatively, rigorous criteria to neglect it must be proposed. In addition, the flame curvature affects both the leading edge and flamelet characteristics in statistically steady incompressible turbulent premixed flames. ${ }^{43}$ Moreover, curvature contributions can dominate diffusion rates and displacement speed for ignition kernels of small radii ${ }^{44,45}$ and determine their probability of survival. Furthermore, the flame curvature is intrinsically related to the analysis of hydro-dynamic and thermo-diffusive instabilities in premixed flames, ${ }^{1-3,46-48}$ and the skewness of curvature probability density function can be related to the Darrieus-Landau instability in premixed turbulent flames. ${ }^{48}$

From the above discussion, it is evident that flame curvature is a key quantity for the analysis and modelling of turbulent premixed combustion. Therefore, in addition to DNS, several experimental analyses focused on flame curvature statistics. However, almost all experimental measurements of curved combustion fronts employed planar imaging (for example, planar laser-induced fluorescence, PLIF) and correspond to 2D curvatures not even in a principal plane of curvature. Shepherd and Ashurst ${ }^{49,50}$ compared planar measurements of curvature and DNS results, showing a reasonable agreement with symmetric PDFs of curvature about zero. Lee et al..$^{51,52}$ obtained PDFs of curvature from OH PLIF and also showed symmetric PDFs of curvature with zero mean value and variance increasing with turbulence intensity. Gashi et al..$^{53}$ obtained and compared PDFs of 2D curvatures of turbulent premixed flame kernels with PLIF of $\mathrm{OH}$ and 3D curvatures via DNS; the limitations of 2D PLIF curvature measurements are quantified by comparison with the 3D DNS information. Yuen and Gülder ${ }^{54}$ investigated premixed methane-air and propane-air turbulent flames stabilized on a Bunsen type burner using planar Rayleigh scattering and Particle Image Velocimetry (PIV); the curvature PDF shows a Gaussian-like behavior at all turbulence intensities for both fuels and at all sections of the flame. Kerl et al. ${ }^{28}$ used a quad-plane PIV technique to investigate the $3 \mathrm{D}$ behavior of flame displacement speed and front curvature of a premixed methane flame stabilized in a diffuser burner; 2D and 3D data are compared to critically assess errors of planar measurements. The statistics of 2D curvature PDFs have been compared to 3D curvature PDFs using DNS data, ${ }^{27,55}$ and it has been found that 2D statistics compare well with the results of planar experimental techniques, but while 2D PDFs are symmetric with nearly zero average mean curvature, $3 \mathrm{D}$ PDFs display positive skewness with negative average mean curvature.

Although the aforementioned analyses identified the pivotal role played by curvature in the statistics of displacement speed and SDF, the physical mechanisms responsible for curvature evolution in turbulent premixed flames are yet to be scrutinized in detail. Dopazo et al. ${ }^{56}$ derived a transport equation for mean curvature in general Cartesian coordinates and computed the various source terms from a DNS database of mixing inert and reacting fluids at constant density in statistically homogeneous, isotropic turbulence. However, no such analysis has been carried out for turbulent premixed flames, in spite of the pivotal role played by curvature in turbulent premixed flame modelling. This gap is addressed in the present work by analyzing the behavior of the iso-surface curvature transport equation in a turbulent lean methane-air bluff body burner configuration based on a flame-resolved high-fidelity simulation database. ${ }^{57}$ The main objectives of this paper are summarized as follows:

- Analysis of the statistics of the various contributions to the curvature evolution equation for a turbulent premixed bluff body burner, which has been analyzed in extensive experiments. ${ }^{58-63}$

- Identification of the leading order physical mechanisms that contribute to the curvature generation.

- Quantifying the values of the terms in the curvature evolution equation in relation to small-scale flame topologies. 


\section{MATHEMATICAL BACKGROUND}

The transport equation of the reaction progress variable $c(\mathbf{x}, t)$ (which increases monotonically from 0 in fresh reactants to 1 in burnt products) takes the following kinematic form for a given $c$-isosurface:

$$
\frac{\partial c}{\partial t}+v_{j}^{c} \frac{\partial c}{\partial x_{j}}=0
$$

In this equation, $v_{j}^{c}$ is the $j$ th component of the local velocity of a point on the iso-surface element. For the present analysis, the reaction progress variable is defined with the combined mass fractions of $\mathrm{CO}_{2}, \mathrm{CO}$, and $\mathrm{H}_{2} \mathrm{O}\left(Y_{c}=Y_{\mathrm{CO}_{2}}+Y_{\mathrm{CO}}+Y_{\mathrm{H}_{2} \mathrm{O}}\right)$ in the following manner: ${ }^{37,57} c=\left[Y_{c}-Y_{c}^{\min }(\xi)\right] /\left[Y_{c}^{\max }(\xi)\right.$ - $Y_{c}^{\min }(\xi)$ ], where $Y_{c}^{\max }(\xi)$ and $Y_{c}^{\min }(\xi)$ are the maximum and minimum values of $Y_{c}$, respectively, for a given mixture fraction $\xi$. The progress variable obeys the transport equation,

$$
\begin{aligned}
\frac{\partial c}{\partial t}+v_{j} \frac{\partial c}{\partial x_{j}} & =\frac{1}{\rho} \frac{\partial}{\partial x_{j}}\left(\rho D \frac{\partial c}{\partial x_{j}}\right)+\dot{\omega}_{c} \\
& =\frac{1}{\rho} \frac{\partial}{\partial x_{N}}\left(\rho D \frac{\partial c}{\partial x_{N}}\right)+D \frac{\partial c}{\partial x_{N}} n_{i, i}+\dot{\omega}_{c} .
\end{aligned}
$$

In Eq. (2), $v_{j}$ is the $j$ th component of the flow velocity, $\rho$ is the fluid density, $D$ is the Fickian molecular diffusivity coefficient for $c$, and $\dot{\omega}_{c}=\dot{\omega} / \rho$ stands for the production of $c$ by chemical reaction. The local unit vector $\mathbf{n}$ points toward the unburned gas side, normal to the iso-surface, and is defined as $n_{i}=-\left(\partial c / \partial x_{i}\right) /|\nabla c|=-c_{, i} /|\nabla c|=c_{, i} /\left(\partial c / \partial x_{N}\right)$, where $x_{N}$ is the coordinate normal to the iso-surface. Here, $n_{i, i} / 2=0.5 \partial n_{i} / \partial x_{i}=0.5\left(k_{1}+k_{2}\right)=k_{m}$ is the mean value of the two principal curvatures $k_{1}$ and $k_{2}$ of the iso-surface and will be referred to as the mean flame curvature. The principal curvatures $k_{1}$ and $k_{2}$ are the eigenvalues of the curvature tensor $\partial n_{i} / \partial x_{j}=n_{i, j}$. In particular, the principal curvatures are given by $k_{1}, k_{2}=\left(-I_{1} \pm \sqrt{I_{1}^{2}-4 I_{2}}\right) / 2$, where $I_{1}=-\partial n_{i} / \partial x_{i}$ and $I_{2}=0.5\left[\left(\partial n_{i} / \partial x_{i}\right)\left(\partial n_{j} / \partial x_{j}\right)-\left(\partial n_{i} / \partial x_{j}\right)\left(\partial n_{j} / \partial x_{i}\right)\right]$ are the first and second invariants of $\partial n_{i} / \partial x_{j}$, respectively. Based on this convention, the flame surface has a positive (negative) curvature where the flame is convex (concave) to the reactants. The term involving $n_{i, i}$ in Eq. (2) is the molecular diffusion rate tangential to the iso-surface.

Equation (2) can be reformulated for a given $c$ isosurface ${ }^{10-20,22-24,26,27,29,31,36}$ as

$$
\frac{\partial c}{\partial t}+v_{j} \frac{\partial c}{\partial x_{j}}=S_{d}|\nabla c|
$$

The local velocity of an iso-surface element is $v_{i}^{c}=v_{i}$ $+S_{d} n_{i}$, where $S_{d}$ is its normal displacement speed relative to the flow. Equating the right-hand sides of Eqs. (2) and (3) yields ${ }^{10-20,22-24,26,27,29,31,36}$

$$
S_{d}(\mathbf{x}, t)=-\frac{1}{\rho\left(\partial c / \partial x_{N}\right)} \frac{\partial}{\partial x_{N}}\left(\rho D \frac{\partial c}{\partial x_{N}}\right)-D n_{j, j}-\frac{\dot{\omega}_{c}}{\left(\partial c / \partial x_{N}\right)} .
$$

The displacement speed $S_{d}$ is the velocity at which the isosurfaces of the reaction progress variable propagate normal to themselves with respect to an initially coincident material surface. The displacement speed in premixed flames is determined by both reaction and molecular diffusion mechanism, whereas in the passive scalar mixing, the effects of reaction are completely absent. The analysis by Dopazo et al. ${ }^{56}$ was done in the context of passive and reactive scalar mixing in a box of constant-density fluid; hence, chemical heat release did not affect the evolution of scalar-gradients and turbulent micro-mixing.

Differentiating Eq. (1) with respect to $x_{i}$ and setting $c, i=-|\nabla c| n_{i}$, equations can be obtained for $|\nabla c|$ and $n_{i},{ }^{62-64}$

$$
\begin{gathered}
\frac{1}{|\nabla c|}\left(\frac{\partial|\nabla c|}{\partial t}+v_{j}^{c} \frac{\partial|\nabla c|}{\partial x_{j}}\right)=-n_{i} v_{j, i}^{c} n_{j}, \\
\frac{\partial n_{i}}{\partial t}+v_{j}^{c} \frac{\partial n_{i}}{\partial x_{j}}=-\left(\delta_{i j}-n_{i} n_{j}\right) S_{j k}^{c} n_{k}+W_{i j}^{c} n_{j} .
\end{gathered}
$$

In Eqs. (5) and (6), $n_{i} v_{j, i}^{c} n_{j}=n_{i} S_{i j}^{c} n_{j}=a_{N}^{c}=a_{N}+\partial S_{d} / \partial x_{N}$ is the total or effective normal strain rate normal to the isosurface, $a_{N}=n_{i} S_{i j} n_{j}$ is the flow normal strain rate, and $\partial S_{d} / \partial x_{N}$ is an added normal strain rate induced by molecular mixing and combustion. ${ }^{64-66}$ The velocity gradient of a surface element can be expressed as

$$
\begin{aligned}
v_{j, i}^{c}= & \frac{\partial v_{j}^{c}}{\partial x_{i}}=\frac{1}{2}\left(\frac{\partial v_{j}^{c}}{\partial x_{i}}+\frac{\partial v_{i}^{c}}{\partial x_{j}}\right)+\frac{1}{2}\left(\frac{\partial v_{j}^{c}}{\partial x_{i}}-\frac{\partial v_{i}^{c}}{\partial x_{j}}\right) \\
= & \frac{1}{2}\left(\frac{\partial v_{j}}{\partial x_{i}}+\frac{\partial v_{i}}{\partial x_{j}}\right)+\frac{1}{2}\left(\frac{\partial v_{j}}{\partial x_{i}}-\frac{\partial v_{i}}{\partial x_{j}}\right) \\
& +\frac{1}{2}\left(n_{j} \frac{\partial S_{d}}{\partial x_{i}}+n_{i} \frac{\partial S_{d}}{\partial x_{j}}\right)+\frac{1}{2}\left(n_{j} \frac{\partial S_{d}}{\partial x_{i}}-n_{i} \frac{\partial S_{d}}{\partial x_{j}}\right) \\
& +S_{d} \frac{1}{2}\left(\frac{\partial n_{j}}{\partial x_{i}}+\frac{\partial n_{i}}{\partial x_{j}}\right)+S_{d} \frac{1}{2}\left(\frac{\partial n_{j}}{\partial x_{i}}-\frac{\partial n_{i}}{\partial x_{j}}\right) .
\end{aligned}
$$

\begin{tabular}{|c|c|}
\hline Description & Term \\
\hline Total strain rate tensor & $S_{i j}^{c}=0.5\left(\partial v_{i}^{c} / \partial x_{j}+\partial v_{j}^{c} / \partial x_{i}\right)$ \\
\hline Total rotation rate tensor & $W_{i j}^{c}=0.5\left(\partial v_{i}^{c} / \partial x_{j}-\partial v_{j}^{c} / \partial x_{i}\right)$ \\
\hline Flow strain rate tensor & $S_{i j}=0.5\left(\partial v_{i} / \partial x_{j}+\partial v_{j} / \partial x_{i}\right)$ \\
\hline Flow rotation rate tensor & $\begin{array}{c}W_{j i}=0.5\left(\partial v_{i} / \partial x_{j}-\partial v_{j} / \partial x_{i}\right) \\
S_{i j}^{a}=0.5\left[\left(\partial S_{d} / \partial x_{j}\right) n_{i}+\left(\partial S_{d} / \partial x_{i}\right) n_{j}\right]\end{array}$ \\
\hline \multirow[t]{2}{*}{ Added strain rate tensor } & $\begin{array}{c}\text { Space dependence of } S_{d} \\
+S_{d} 0.5\left(\partial n_{i} / \partial x_{j}+\partial n_{j} / \partial x_{i}\right)\end{array}$ \\
\hline & $W_{i j}^{a}=\underbrace{0.5\left[\left(\partial S_{d} / \partial x_{j}\right) n_{i}-\left(\partial S_{d} / \partial x_{i}\right) n_{j}\right]}_{\text {Propagating curved iso-c }}$ \\
\hline \multirow[t]{2}{*}{ Added rotation rate tensor } & $\begin{array}{c}\text { Space dependence of } S_{d} \\
+S_{d} 0.5\left(\partial n_{i} / \partial x_{j}-\partial n_{j} / \partial x_{i}\right)\end{array}$ \\
\hline & Propagating curved iso-c \\
\hline
\end{tabular}

The nomenclature associated with terms on the right-hand side of Eq. (7) is summarized in Table I, where the total strain rate and rotation rate tensors are expressed as $S_{i j}^{c}=S_{i j}+S_{i j}^{a}$ and $W_{i j}^{c}=W_{i j}+W_{i j}^{a}$, respectively. ${ }^{64-66}$

The flow strain rate and rotation rate tensors $S_{i j}$ and $W_{i j}$ are due to the fluid motion, and the added strain rate and rotation rate tensors $S_{i j}^{a}$ and $W_{i j}^{a}$ are due to the motion of curved iso-surfaces relative to the flow with spatially dependent $S_{d}$.

TABLE I. Nomenclature associated with the velocity gradient $v_{i, j}^{c}$ of an isosurface element. 
TABLE II. Description of the various terms in the mean curvature transport equation (8).

\begin{tabular}{|c|c|c|c|}
\hline Terms & Description & Terms & Description \\
\hline$T_{1}$ & Contribution due to curvature/flow normal strain rate & $T_{6}$ & Contribution due to curvature/added normal strain rate \\
\hline$T_{2}$ & $\begin{array}{l}\text { Contribution due to flow normal strain rate variation } \\
\text { in flame normal direction }\end{array}$ & $T_{7}$ & $\begin{array}{l}\text { Contribution due to added normal strain rate variation } \\
\text { in flame normal direction }\end{array}$ \\
\hline$T_{3}$ & Curvature flow stretching term & $T_{8}$ & Curvature added flow stretching term \\
\hline$T_{4}$ & Contribution due to flow strain rate gradients & $T_{9}$ & Contribution due to added strain rate gradients \\
\hline$T_{5}$ & Contribution due to the flow vorticity curl & $T_{10}$ & Contribution due to the added vorticity curl \\
\hline
\end{tabular}

The first term on the right-hand side of Eq. (6) for $n_{i}$ is the projection of the strain vector $-\mathbf{S}^{c} \cdot \mathbf{n}$ on a plane perpendicular to $\mathbf{n}$, whereas the second term $W_{i j}^{c} n_{j}=\frac{1}{2} \varepsilon_{i j k} \omega_{j}^{c} n_{k}$ is the solid body rotation of $\mathbf{n}$ with an angular velocity $\frac{1}{2} \omega^{c}$ (the total vorticity vector $\omega^{c}=\nabla \times \mathbf{v}^{c}=\nabla \times \mathbf{v}+\nabla \times\left(S_{d} \mathbf{n}\right)$ at a point on the iso-surface is the sum of the flow vorticity $\omega=\nabla \times \mathbf{v}$ and the added vorticity $\left.\boldsymbol{\omega}^{a}=\nabla \times\left(S_{d} \mathbf{n}\right)=\nabla S_{d} \times \mathbf{n}+S_{d} \nabla \times \mathbf{n}\right)$. Both contributions are perpendicular to $\mathbf{n} .{ }^{56}$

Taking the derivative of Eq. (6) for $n_{i}$ with respect to $x_{i}$ yields an evolution equation for the mean curvature $k_{m},{ }^{56}$

$$
\begin{aligned}
& \frac{\partial k_{m}}{\partial t}+v_{j}^{c} \frac{\partial k_{m}}{\partial x_{j}} \\
& =\underbrace{\underbrace{\frac{a_{N} n_{i, i}}{2}}_{T_{1}}+\underbrace{\frac{1}{2} \frac{\partial a_{N}}{\partial x_{N}}}_{T_{2}} \underbrace{-S_{i j} n_{j, i}}_{T_{3}} \underbrace{\frac{1}{2} \frac{\partial S_{i j}}{\partial x_{i}} n_{j}}_{T_{4}}+\underbrace{\frac{1}{2} \frac{\partial W_{i j}}{\partial x_{i}} n_{j}}_{T_{5}}}_{\text {flow terms }} \\
& +\underbrace{\underbrace{\frac{1}{2} \frac{\partial S_{d}}{\partial x_{N}} n_{i, i}}_{T_{6}}+\underbrace{\frac{1}{2} \frac{\partial^{2} S_{d}}{\partial x_{N} 2}}_{T_{7}} \underbrace{-S_{i j}^{a} n_{j, i}}_{T_{8}} \underbrace{-\frac{1}{2} \frac{\partial S_{i j}^{a}}{\partial x_{i}} n_{j}}_{T_{9}}+\underbrace{\frac{1}{2} \frac{\partial W_{i j}^{a}}{\partial x_{i}} n_{j}}_{T_{10}}}_{\text {added terms }} .
\end{aligned}
$$

The right-hand side of Eq. (8) shows (i) the terms $T_{1-5}$ arising from the fluid flow and (ii) the terms $T_{6-10}$ added by molecular mixing and combustion. The positive terms on the right-hand side of Eq. (8) are sources of mean curvature and tend to increase the convexity of iso-surfaces; negative terms are sinks that decrease the convexity. The physical meaning of the terms in Eq. (8) is summarized in Table II.

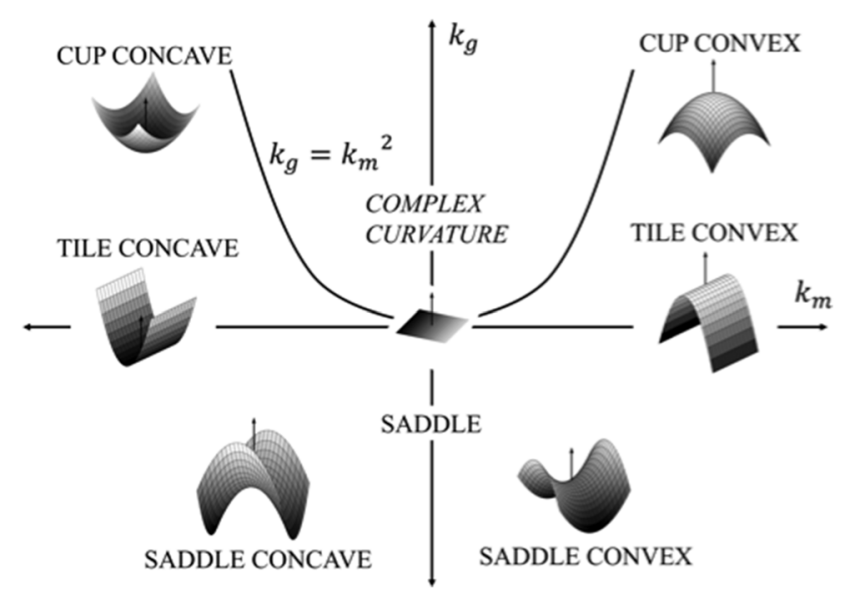

FIG. 1. Classification of iso-surface geometries in the plane of mean $k_{m}$ and Gauss curvature $k_{g} .{ }^{67}$
The terms $T_{5}$ and $T_{10}$ in Eq. (8) can be recast as ${ }^{56}$

$$
\begin{gathered}
T_{5}=\frac{1}{2} \frac{\partial W_{i j}}{\partial x_{i}} n_{j}=\frac{1}{4} n_{i} \varepsilon_{i j k} \frac{\partial \omega_{k}}{\partial x_{j}} \\
T_{10}=\frac{1}{2} \frac{\partial W_{i j}^{a}}{\partial x_{i}} n_{j}=\frac{1}{4} n_{i} \varepsilon_{i j k} \frac{\partial \omega_{k}^{a}}{\partial x_{j}}=-\frac{1}{4}\left(\delta_{i j}-n_{i} n_{j}\right) \frac{\partial^{2} S_{d}}{\partial x_{i} \partial x_{j}} \\
+\frac{1}{4}\left(\frac{\partial S_{d}}{\partial x_{N}} \frac{\partial n_{j}}{\partial x_{j}}+\frac{\partial S_{d}}{\partial x_{j}} \frac{\partial n_{j}}{\partial x_{N}}\right)-\frac{1}{4} S_{d} n_{i} \frac{\partial}{\partial x_{j}}\left(\frac{\partial n_{i}}{\partial x_{j}}-\frac{\partial n_{j}}{\partial x_{i}}\right) .
\end{gathered}
$$

Results for the terms $T_{1-10}$ are presented after normalisation $T_{\alpha}^{*}=T_{\alpha}\left(\delta_{L}^{2} / S_{L}\right)$ and conditioned on small-scale flame geometries later in this paper. These local iso-scalar geometries can be characterized in terms of the mean curvature $k_{m}=0.5\left(k_{1}+k_{2}\right)$ and the Gauss curvature $k_{g}=k_{1} k_{2}$, as shown in Fig. $1 .{ }^{67}$ The region of complex curvatures cannot be realized in practice. The small-scale geometries associated with $k_{g}>0$ correspond to cup-convex and cup-concave structures for $k_{m}>0$ and $k_{m}<0$, respectively. The structures with $k_{g}<0$ lead to saddle-like geometries, which can be either positively (i.e., $k_{m}>0$ ) or negatively (i.e., $k_{m}<0$ ) curved. A zero value of $k_{g}$ leads to tile structures, either tile convex or tile concave for $k_{m}>0$ or $k_{m}<0$. Figure 1 will subsequently be used in this paper to facilitate the discussion related to the iso-surface geometries in the $k_{m}-k_{g}$ plane. ${ }^{67}$ The results are presented in normalized form, for $k_{m}^{*}=k_{m} \delta_{L}$ and $k_{m}^{*}=k_{m} \delta_{L}^{2}$, where $\delta_{L}$ is the laminar flame thickness $\delta_{L}=\left(T_{a d}-T_{u}\right) / \max |\nabla T|_{L}$. The subindex $L$ refers to unstrained laminar flame values.

\section{NUMERICAL IMPLEMENTATION}

The flame resolved simulation has been described by Sandeep et al. ${ }^{37}$ and Proch et al. ${ }^{57}$ in detail, and only a brief summary is provided here. The Cambridge stratified flame has been simulated by various groups applying Reynolds Averaged Navier-Stokes simulations (RANS), LES, ${ }^{68-70}$ and the highly resolved simulation of the present case. Experimental data were provided by Hochgreb and Barlow and co-workers, ${ }^{58-63}$ leading to the unique situation that "flame DNS" data could be validated against a real flame experiment.

The burner consists of a bluff body surrounded by two co-annular methane-air streams with an equivalence ratio of $\phi=0.75$ at atmospheric conditions, embedded in an air-coflow with a velocity of $0.4 \mathrm{~m} / \mathrm{s}$, as illustrated in Fig. 2. The Lewis number was unity for all species, and the chemistry was tabulated with the Premixed Flamelet Generated Manifold (PFGM) approach. ${ }^{71-73}$ The Kolmogorov micro-scale is $\eta=41 \mu \mathrm{m}$ at the inlet and increases significantly downstream and from the 


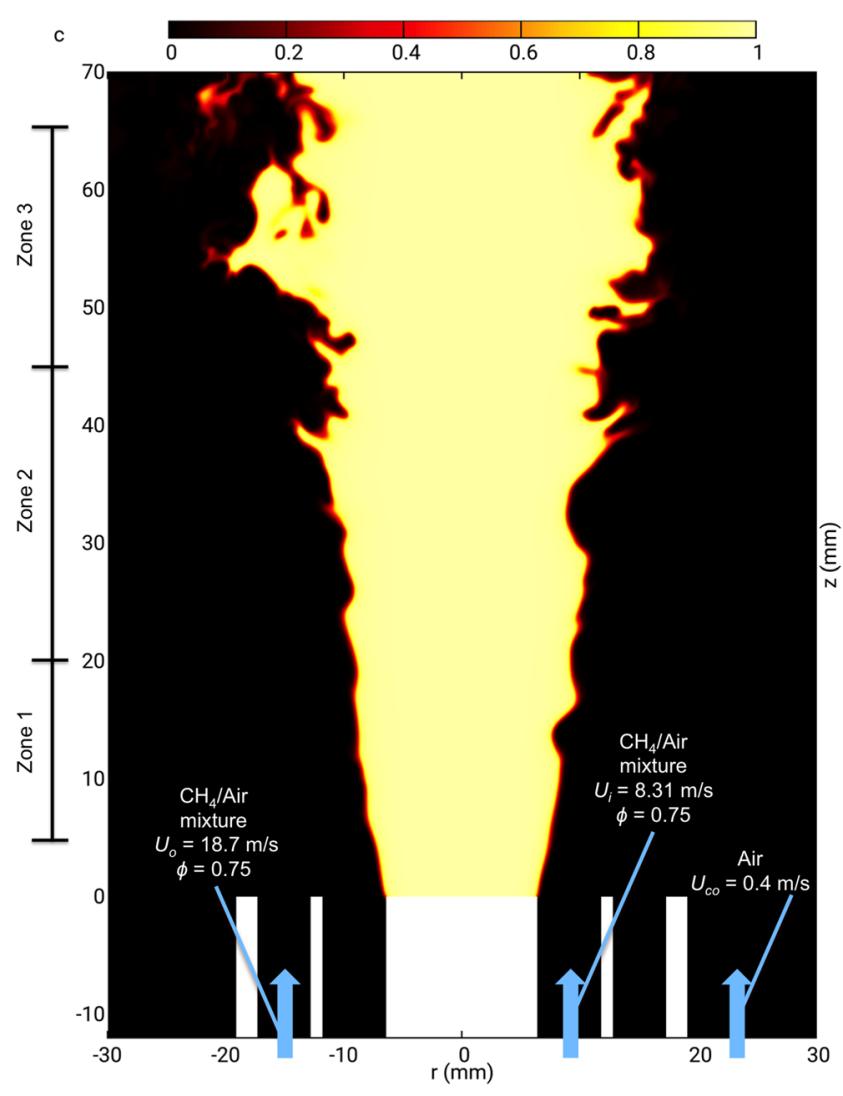

FIG. 2. Instantaneous progress variable $c$ in the burner mid-section.

unburned to the burned side of the flame. The ratio of the grid resolution to the Kolmogorov micro-scale in the reaction zone is $\Delta / \eta \approx 1$, which means that the velocities scales are resolved. The results are analyzed at $t=0.34 \mathrm{~s}$, many initial integral eddy turnover times $\left(\tau=60 \times 10^{-5} \mathrm{~s}\right.$ based on the inner stream mean velocity) into the simulation, and after one flow-through time $(t=0.28 \mathrm{~s})$ of the slow air co-flow. Table III gives the inlet flow velocities of the co-annular streams $U$, the integral length scale $l$, the root-mean-square turbulent velocity fluctuation $u^{\prime}$, the unstrained laminar burning velocity $S_{L}$, the Damköhler number $D a=l S_{L} / u^{\prime} \delta_{L}$, the Karlovitz number $K a=\left(u^{\prime} / S_{L}\right)^{3 / 2}\left(l / \delta_{L}\right)^{-1 / 2}$, and the turbulent Reynolds number $R e_{t}=u^{\prime} l / v$, where the kinematic viscosity $v$ depends on the temperature. The statistics have been evaluated at different axial locations above the burner, namely, in Zone 1 (5-20 mm), Zone $2(20-45 \mathrm{~mm})$, and Zone $3(45-65 \mathrm{~mm})$ as illustrated in Fig. 2. The value of $u^{\prime} / S_{L}$ increases, whereas $l / \delta_{L}$ does not change appreciably with increasing axial distance. ${ }^{57}$ This suggests that $K a=\left(u^{\prime} / S_{L}\right)^{3 / 2}\left(l / \delta_{L}\right)^{-1 / 2}$ increases in the downstream direction and combustion takes place at the boundary of the corrugated flamelets and thin reaction zone regimes of combustion $^{74}$ close to the inlet, whereas combustion takes place well within the thin reaction zone regime away from the nozzle inlet. Interested readers are referred to Fig. 15 of Ref. 57 for further information in this regard.

\section{RESULTS AND DISCUSSIONS}

\section{A. Terms of the curvature evolution equation conditional upon the progress variable}

Figure 3 shows the mean values of the terms in the curvature transport equation (8), conditioned on the reaction progress variable $c$ for the three axial locations (Zone 1: 5-20 mm, Zone 2: 20-45 mm, and Zone 3: 45-65 mm). The ensemble averages were extracted from the given iso-surfaces at $t=0.34 \mathrm{~s}$, as in previous analyses. ${ }^{16-20,55,75}$ The flame width changes significantly in the axial direction (see Figs. 9 and 19 of Ref. 57) and thus plotting the terms conditional on $c$ is more sensible for the purpose of comparison of the curvature transport terms between different zones. It is apparent that some of the added terms arising from flame propagation (see Table II) are one or two orders of magnitude greater than most flow contributions, depending on the axial location in the flame, as illustrated in Fig. 3(c).

The mean values of the flow terms $T_{1-5}$ conditioned on $c$ increase with the axial height as the flame wrinkling increases downstream. Terms $T_{2}, T_{3}$, and $T_{4}$ seem to be the dominant. The mean value of the term due to curvature and the flow normal strain rate, $T_{1}^{*}=T_{1}\left(\delta_{L}^{2} / S_{L}\right)=\left[\left(a_{N} n_{i, i}\right) / 2\right]\left(\delta_{L}^{2} / S_{L}\right)$, assumes weak positive values for $c>0.3$ near the burner and becomes increasingly negative downstream; the latter implies that $a_{N}>0$ is predominantly associated with concave local structures $\left(k_{m}<0\right)$, while $a_{N}<0$ occurs for convex topologies $\left(k_{m}>0\right)$. It has been demonstrated earlier that flame wrinkling increases further away from the burner where some turbulent eddies penetrate the preheat region, thereby increasing the magnitude of curvature $k_{m}=n_{i, i} / 2$. The turbulent straining dominates over the flame normal acceleration, ${ }^{15,31}$ as the axial distance from the burner increases, which gives rise to a preferential alignment of $\nabla c$ with the eigenvector associated with the most compressive principal strain rate. ${ }^{37,57,76}$ Thus, the share of negative values of $a_{N}$ increases downstream (not shown here), ${ }^{37}$ and the negative values of $a_{N}$ are more frequent for convex $\left(k_{m}>0\right)$ flame topologies. ${ }^{77}$ Furthermore, the effects of dilatation are strong in the negatively curved regions ${ }^{17,18,26,37}$ because of the focusing of heat, and this increases the extent of $\nabla c$ alignment with the most extensive principal strain rate, which produces locally either positive or small negative values of $a_{N}$. The negative correlation between $a_{N}$ and $k_{m}{ }^{37}$ leads to the negative mean values of $T_{1}^{*}$ away from the nozzle exit.

TABLE III. Parameters of the inner and outer reactant streams.

\begin{tabular}{lccccccccc}
\hline \hline Stream & $l(\mathrm{~mm})$ & $u^{\prime}(\mathrm{m} / \mathrm{s})$ & $S_{L}(\mathrm{~m} / \mathrm{s})$ & $\delta_{L}(\mathrm{~mm})$ & $R e(-)$ & $\phi(-)$ & $D a(-)$ & $K a(-)$ & $R e_{t}(-)$ \\
\hline Inner & 0.5 & 0.9 & 0.21 & 0.56 & 5960 & 0.75 & 0.2 & 9.3 & 28.1 \\
Outer & 0.5 & 1.8 & 0.21 & 0.56 & 11500 & 0.75 & 0.1 & 26.3 & 56.3 \\
\hline
\end{tabular}

$U_{i}=8.31 \mathrm{~m} / \mathrm{s}, U_{0}=18.7 \mathrm{~m} / \mathrm{s}$, and $U_{c o}=0.4 \mathrm{~m} / \mathrm{s}$ 


$$
\begin{aligned}
& \cdots T_{1}^{*} \quad 0 \quad T_{3} \\
& \text { 一 } T_{2}^{*} \quad \text { অㅁ. } T_{4}^{*}
\end{aligned}
$$<smiles>[123I-]</smiles>

(a)
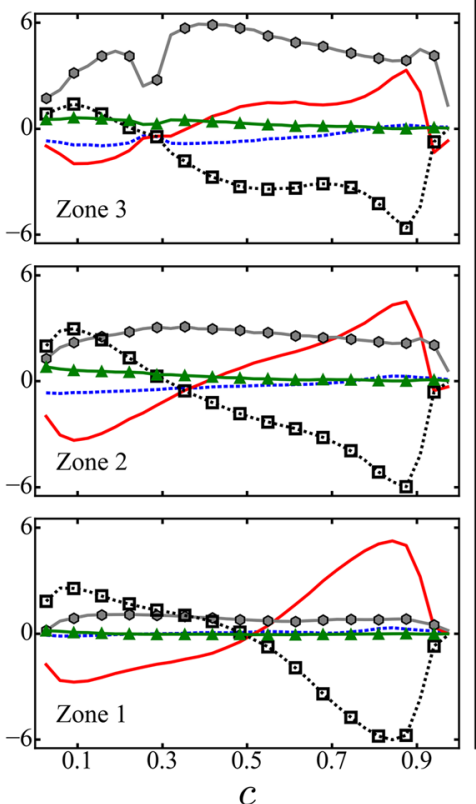

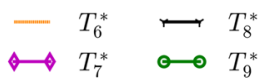

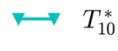

(b)
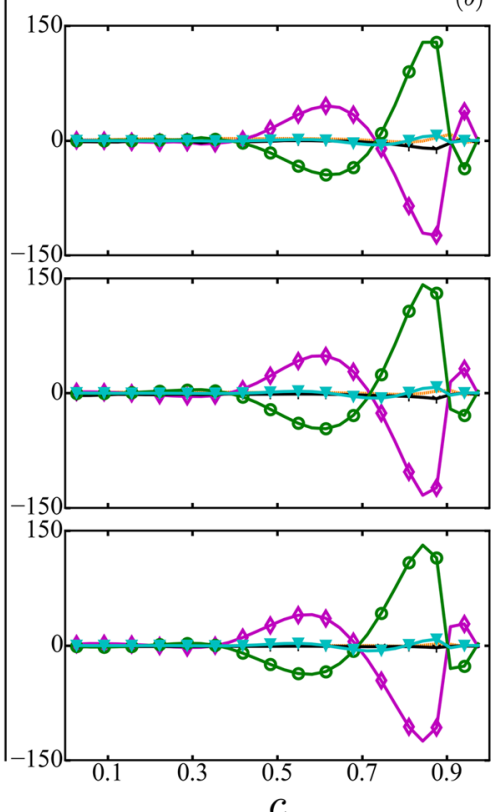

….. $T_{1}^{*} \longmapsto T_{5}^{*} \longrightarrow T_{8}^{*}$

$\longrightarrow T_{2}^{*}-T_{6}^{*} \multimap T_{9}^{*} / 10$

$\longleftrightarrow T_{3}^{*} \longleftrightarrow T_{7}^{*} / 10 \longmapsto T_{10}^{*}$

a… $T_{4}^{*}$

(c)

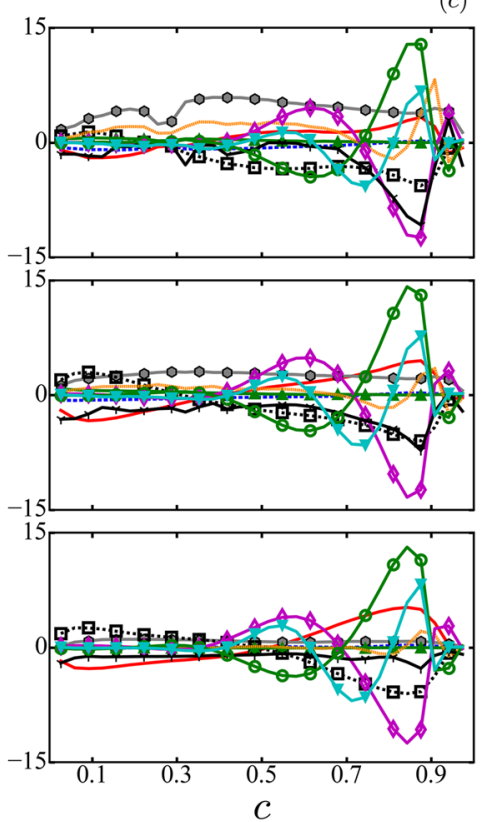

FIG. 3. Average values of the mean curvature transport terms of Eq. (8), conditioned on the reaction progress variable $c$ at different axial locations. (a) Flow terms, (b) added terms, and (c) all terms; the mean values of $T_{7}^{*}$ and $T_{9}^{*}$ have been scaled by ten. Results are separately examined in three zones (Zone 1 : 5-20 mm, Zone 2: 20-45 mm, and Zone 3: 45-65 mm are shown in 3rd, 2nd, and 1st rows, respectively).

The mean value of $T_{2}^{*}=T_{2}\left(\delta_{L}^{2} / S_{L}\right)=\left[\left(\partial a_{N} / \partial x_{N}\right) / 2\right]$ $\left(\delta_{L}^{2} / S_{L}\right)$, which is due to the normal gradients of the flow normal strain rate, assumes negative values on the unburnt side, shifting toward positive values in the reaction layer and retaining this behaviour in different zones above the burner exit. This is consistent with the dependence of $a_{N}$ on $x_{N}$ in this flame; ${ }^{37,57}$ $a_{N}$ increases from the fresh reactants $\left(\partial a_{N} / \partial x_{N}<0\right)$, reaches a maximum within the flame, and decreases toward the hot products $\left(\partial a_{N} / \partial x_{N}>0\right)$. Thermal expansion due to heat release within the reaction zone promotes the alignment of $\nabla c$ with the most extensive principal strain rate and yields a maximum of $a_{N}$ in the reaction zone.

The mean value of the curvature flow stretching term $T_{3}^{*}=T_{3}\left(\delta_{L}^{2} / S_{L}\right)=-\left(S_{i j} n_{j, i}\right)\left(\delta_{L}^{2} / S_{L}\right)$ assumes mostly positive values, with a significant increase in magnitude in the downstream direction. Using the principal axes tangent to a local isosurface $\left(\mathrm{x}_{T 1}, \mathrm{x}_{T 2}\right)$, the curvature flow stretching term can be written as $T_{3}=-\left(S_{11} k_{1}+S_{22} k_{2}\right)$, where $S_{11}$ and $S_{22}$ are the unitary tangential strain rates along axes $\mathrm{x}_{T 1}$ and $\mathrm{x}_{T 2}$, respectively, and $k_{1}$ and $k_{2}$ are the principal curvatures. It is worth noting that $a_{T}=S_{11}+S_{22}$ and $a_{N}$ may predominantly assume positive values when $\nabla c$ predominantly aligns with the eigenvector associated with the most extensive principal strain rate. ${ }^{37,57,76}$ A positive value of $T_{3}$ implies a combination of elliptic and hyperbolic scalar topologies ${ }^{67}$ with positive and negative values of $k_{1}$ and $k_{2}$. For example, an elliptic concave structure $\left(k_{1}<0\right.$ and $\left.k_{2}<0\right)$ yields $T_{3}>0$ for a combination of $S_{11}>0$ and $S_{22}>0$.

The profile of the mean value of the term $T_{4}^{*}$ $=-\left[\left(\partial S_{i j} / \partial x_{i}\right)\left(n_{j} / 2\right)\right]\left(\delta_{L}^{2} / S_{L}\right)$ conditional upon $c$ is similar to that of $-T_{2}^{*}$. This means that the two vectors $n_{j}$ and $\partial S_{i j} / \partial x_{i}$ point in the same direction within the flame but tend to point in opposite directions on the unburned side of the flame.

The mean contribution to the curvature evolution due to vorticity gradients, $T_{5}^{*}=T_{5}\left(\delta_{L}^{2} / S_{L}\right)=\left[\left(\partial W_{i j} / \partial x_{i}\right)\left(n_{j} / 2\right)\right]$ $\left(\delta_{L}^{2} / S_{L}\right)$, can be rewritten as $T_{5}^{*}=\left\{\left(n_{i} / 4\right)\left[\varepsilon_{i j k}\left(\partial \omega_{k} / \partial x_{j}\right)\right]\right\}$ $\left(\delta_{L}^{2} / S_{L}\right)$; in vector notation, $T_{5}=[\mathbf{n} \cdot(\nabla \times \omega)] / 4$ [see Eq. (9)]. For example, on an iso-surface and using the local principal axes $\left(\mathrm{x}_{T 1}, \mathrm{x}_{T 2}, \mathrm{x}_{N 3}\right), T_{5}$ can be expressed as $T_{5}=\left(\partial \omega_{2} / \partial x_{1}\right.$ $\left.-\partial \omega_{1} / \partial x_{2}\right) / 4$ for $\mathbf{n}=(0,0,1)$, where $\omega_{1}$ and $\omega_{2}$ are the components of the flow vorticity vector tangent to the $c$ iso-surface. It can readily be seen that co-rotating parallel vortices of different intensity and counter-rotating parallel vortices of the same intensity can curve a planar local iso-surface structure, fostering positive or negative curvatures. The mean value of $T_{5}$ shifts from small negative values close to the burner to positive values further downstream. The flame-turbulence interaction is weak in Zone $1,{ }^{37,57}$ but some vortical structures penetrate the preheat region in Zones 2 and 3, which increases the spatial variation of vorticity, in turn increasing the magnitude of $T_{5}$ toward the unburned gas side and in the downstream direction. The mean contribution by vorticity gradients $T_{5}^{*}$ remains mostly negligible close to $c=1.0$ due to the annihilation of enstrophy by dilatation and the high dynamic viscosity in the hot products.

The mean values of the added terms due to flame propagation $T_{6}^{*}, T_{8}^{*}$, and $T_{10}^{*}$ have negligible values in comparison to those of the terms $T_{7}^{*}$ and $T_{9}^{*}$ through most of the flame brush. The mean contributions of $T_{7}^{*}$ and $T_{8}^{*}$ exhibit similar trends toward the burned gas side of the flame in Zones 
2 and 3 [see Fig. 3(c)]. High values of $k_{m}=n_{i, i} / 2$ are less probable to occur in Zone 1 since the flame propagates in a low turbulence environment. This behaviour explains the small mean contributions to the curvature evolution in Zone 1 due to the source term of curvature and added normal strain rate $T_{6}^{*}=\left[\left(\partial S_{d} / \partial x_{N}\right)\left(n_{i, i} / 2\right)\right]\left(\delta_{L}^{2} / S_{L}\right)$ and due to the added flow stretching term $T_{8}^{*}=-\left(S_{i j}^{a} n_{j, i}\right)\left(\delta_{L}^{2} / S_{L}\right)$. The term $T_{6}^{*}$ is associated with scalar topologies corresponding to compressive and extensive added normal strain rates.

The term $T_{7}^{*}=T_{7}\left(\delta_{L}^{2} / S_{L}\right)=\left[\left(\partial^{2} S_{d} / \partial x_{N}^{2}\right) / 2\right]\left(\delta_{L}^{2} / S_{L}\right)$ results from the flame normal gradient of the added normal strain rate. The mean variation of $\partial S_{d} / \partial x_{N}$ conditioned upon $c$ has been shown for this case before ${ }^{37}$ and is not repeated here. In the present case, the mean value of $\partial S_{d} / \partial x_{N}$ assumes predominantly negative values for the major part of the flame front before assuming weak positive values on the burned gas side. By contrast, in Zones 2 and 3, the mean value of $\partial S_{d} / \partial x_{N}$ remains positive on both unburned gas and burned gas sides of the flame front and negative values are only obtained in the reaction zone (i.e., $0.6<c<0.9$ ). Accordingly, the mean values of $T_{7}^{*}$ are monotonically increasing from the unburned gas side toward the preheat region taking negative values in the region with high chemical activity and positive on the burned gas side; $T_{7}^{*}$ changes sign from the leading edge to the preheat/burning region to the trailing edge of the flame.

The mean contribution to the added stretching term, $T_{8}^{*}=T_{8}\left(\delta_{L}^{2} / S_{L}\right)=-\left(S_{i j}^{a} n_{j, i}\right)\left(\delta_{L}^{2} / S_{L}\right)$, exhibits small magnitudes in Zone 1, since high values of $n_{i, i}$ are less probable to occur in Zone 1 where the flame propagates in a low turbulence environment. The mean values of $T_{8}^{*}$, conditional on $c$, increase in the downstream direction and attain significant peak values in the reaction zone of the flame. For example, on an iso-surface and using the local principal axes $\left(\mathrm{x}_{T 1}, \mathrm{x}_{T 2}\right.$, $\left.\mathrm{x}_{N 3}\right), T_{8}$ takes the form $T_{8}=-S_{d}\left(k_{1}^{2}+k_{2}^{2}\right)$, for $\mathbf{n}=(0,0$, $1)$, which accordingly yields a negative value in the reaction zone.

The mean contribution due to the added strain gradients $T_{9}^{*}=T_{9}\left(\delta_{L}^{2} / S_{L}\right)=-\left[\left(\partial S_{i j}^{a} / \partial x_{i}\right)\left(n_{j} / 2\right)\right]\left(\delta_{L}^{2} / S_{L}\right)$ depends on the second derivatives of $S_{d}$ and of $n_{i}$ and on cross products of the gradients of $S_{d}$ and the curvature tensor; the profile of the mean value of this term conditional upon $c$ is similar to that of $-T_{7}^{*}$. The mean value of the added vorticity curl contribution $T_{10}^{*}=T_{10}\left(\delta_{L}^{2} / S_{L}\right)$ exhibits a small magnitude in Zone 1 and increases in the downstream direction with significant positive peak values in the reaction zone of the flame of this flow configuration.

\section{B. Terms of the curvature evolution equation conditional upon the flame curvature}

The mean flow contributions $T_{1-5}^{*}$ and the added contributions $T_{6-10}^{*}$ to the mean curvature evolution conditioned upon the normalised mean curvature $k_{m}^{*}=k_{m} \delta_{L}$ are shown in Fig. 4. It can be seen that the added contributions dominate over the flow contributions for all values of $k_{m}^{*}$, which agrees with the results observed in Fig. 3. No clear trend exists between $k_{m}^{*}$ and the flow terms in Zone 1. The flow does not influence small-scale scalar structures in this zone

$$
\begin{array}{cccc}
\cdots \cdots & T_{1}^{*} & 0 & T_{3} \\
- & T_{2}^{*} & \mathbf{\square} \cdots \mathbf{\square} & T_{4}
\end{array}
$$

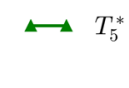

(a)
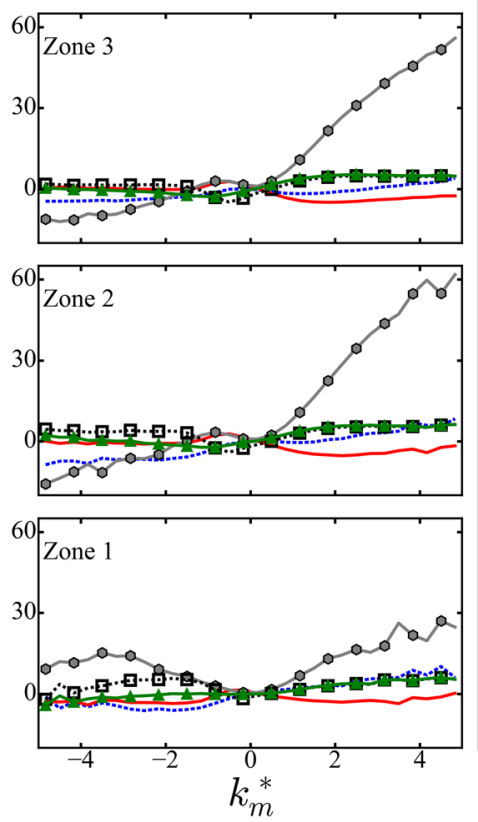

$$
\longrightarrow T_{6}^{*} \longrightarrow T_{8}^{*}
$$
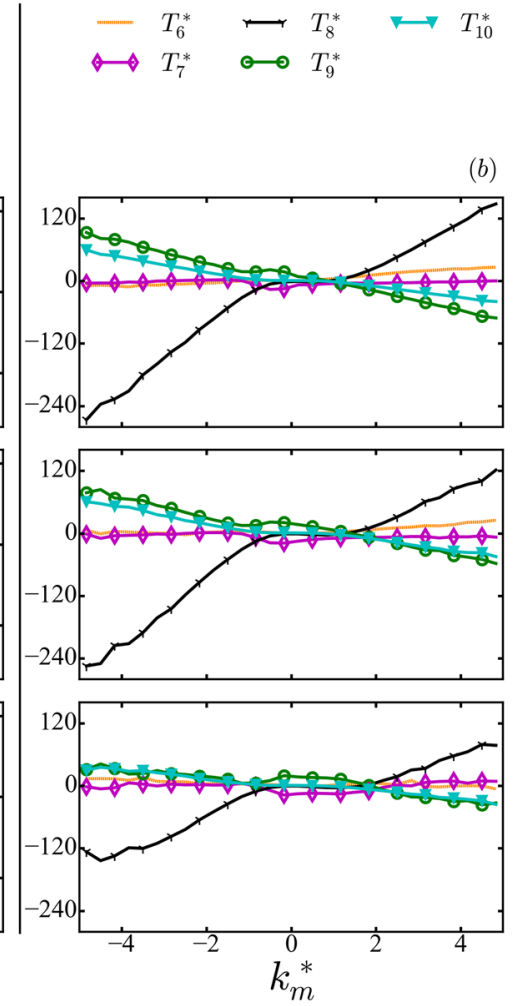

$$
\begin{aligned}
& \cdots T_{1}^{*} \longmapsto T_{5}^{*} \longrightarrow T_{8}^{*} / 2 \\
& \text { — } T_{2}^{*} \longrightarrow T_{6}^{*} \multimap T_{9}^{*} / 2 \\
& \longleftrightarrow T_{3}^{*} \longleftrightarrow T_{7}^{*} \longmapsto T_{10}^{*} / 2 \\
& \text { ㅁㅁ } T_{4}^{*}
\end{aligned}
$$
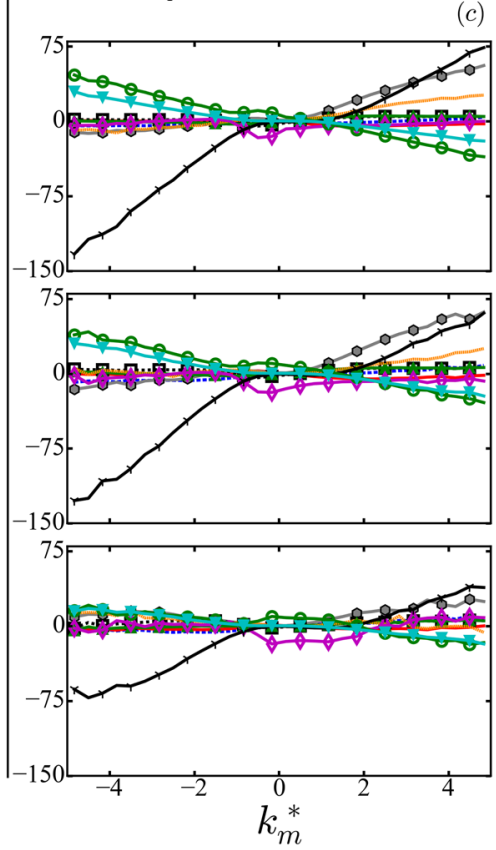

FIG. 4. Average values of the mean terms in the curvature transport equation (8), conditioned upon the mean curvature $k_{m}^{*}$. (a) Flow terms, (b) added terms, and (c) all terms; the mean values of $T_{8}^{*}, T_{9}^{*}$, and $T_{10}^{*}$ have been scaled by two. Results are separately examined in three zones (Zone 1: 5-20 mm, Zone 2: 20-45 mm, and Zone 3: $45-65 \mathrm{~mm}$ are shown in 3rd, 2nd, and 1st rows, respectively). 
due to thd weak flame-turbulence interaction. In Zones 2 and 3 , negative values of $T_{1}^{*}=T_{1}\left(\delta_{L}^{2} / S_{L}\right)=\left[\left(a_{N} n_{i, i}\right) / 2\right]\left(\delta_{L}^{2} / S_{L}\right)$ are related to $k_{m}^{*}<0$, whereas $T_{1}^{*}>0$ correspond to $k_{m}^{*}>0$; it is worth stressing that different iso-surfaces are analyzed in these averages, and since the flow normal strain rate $a_{N}$ is predominantly negative downstream, ${ }^{37}$ the variation of $T_{1}^{*}$ is determined by the mean curvature $k_{m}=n_{i, i} / 2$. On the other hand, $T_{2}^{*}$ seems to be approximately constant for every scalar geometry since there is no correlation between $T_{2}^{*}$ and $k_{m}^{*}$. The term $T_{3}^{*}=T_{3}\left(\delta_{L}^{2} / S_{L}\right)=-\left(S_{i j} n_{j, i}\right)\left(\delta_{L}^{2} / S_{L}\right)$ acts as the leading flow term for topologies with large curvatures, both concave and convex. The profiles of $T_{4}^{*}$ and $T_{5}^{*}$ across the mean curvature are similar. These terms have negligible values toward negative $k_{m}$ for increasing positive values of mean curvature, indicating that gradients of flow strain and rotation rates tend to align with the normal vector $\mathbf{n}$ for convex scalar micro-structures.

The mean values conditioned on curvature for $T_{6}^{*}$ and $T_{7}^{*}$ remain negligible across the flame-front in every zone. The curvature added flow stretching term $T_{8}^{*}=T_{8}\left(\delta_{L}^{2} / S_{L}\right)$ exhibits a positive correlation with $k_{m}$; it has already been mentioned in Sec. IV A that an approximation in principal axes of curvature yields $T_{8}=-S_{d}\left(k_{1}^{2}+k_{2}^{2}\right)$, which implies a cubic dependence on $k_{m}$ accounting for the dependency of $S_{d}$ on mean curvature, so that $T_{8}$ shows an antisymmetric behavior with respect to $k_{m}=0$, with its negative values correlating with $k_{m}<0$, positive values corresponding to $k_{m}>0$, and $k_{m}=0$ being the inflection point. The mean contribution due to the added strain gradients $T_{9}^{*}$ and the mean value of the added vorticity curl contribution $T_{10}^{*}$ display negative correlations with $k_{m}$, which indicate that the significant positive values correlated with the reaction zone of the flame are mainly associated with concave iso-scalar surfaces; these negative correlations are also observed in previous studies between the local volumetric dilatation rate and the mean flame curvature. ${ }^{17,78}$

\section{Flow, added, and total contributions to the curvature evolution}

The mean values of flow contributions $\left(T_{1}^{*}+\cdots+T_{5}^{*}\right)$, the flame propagation induced added contributions $\left(T_{6}^{*}+\cdots+T_{10}^{*}\right)$, and the total contribution $\left(T_{1}^{*}+\cdots+T_{10}^{*}\right)$ to the curvature evolution, conditioned on the reaction progress variable $c$ and the mean curvature $k_{m}^{*}$, for the different zones of the computational domain are shown in Fig. 5.

The overall contributions conditioned on $c$ are positive (convexity increases, concavity decreases) in the unburned gas and the preheat regions and become negative (convexity decreases, concavity increases) in the reaction zone of the flame with positive values in the burnt products. This implies that the positive mean contributions of $T_{3}^{*}$ and $T_{4}^{*}$ dominate over the flame propagation induced added contributions in the unburned gases. On the contrary, $T_{7}^{*}$ is the leading term in the reaction zones and in the burned gas side of the flame front. The total contribution to the $k_{m}$ evolution is controlled by flow terms on the unburned gas side (i.e., $c<0.5$ ) and by the flame propagation-induced added terms for $c>0.5$. The mean overall flow contribution conditioned on $k_{m}^{*}$ is negligible in comparison with the overall added contribution for negative values of $k_{m}^{*}$ but assumes non-negligible values for $k_{m}^{*}>0$. Added flow terms are negligible compared with the flow terms for $k_{m}^{*}>0$ in Zone 1. In Zones 2 and 3, the magnitude of the flow terms increases for $k_{m}^{*}>0$. It can also be seen that the overall mean added contribution arises mainly from the term $T_{8}^{*}$, due to curvature added flow stretching.
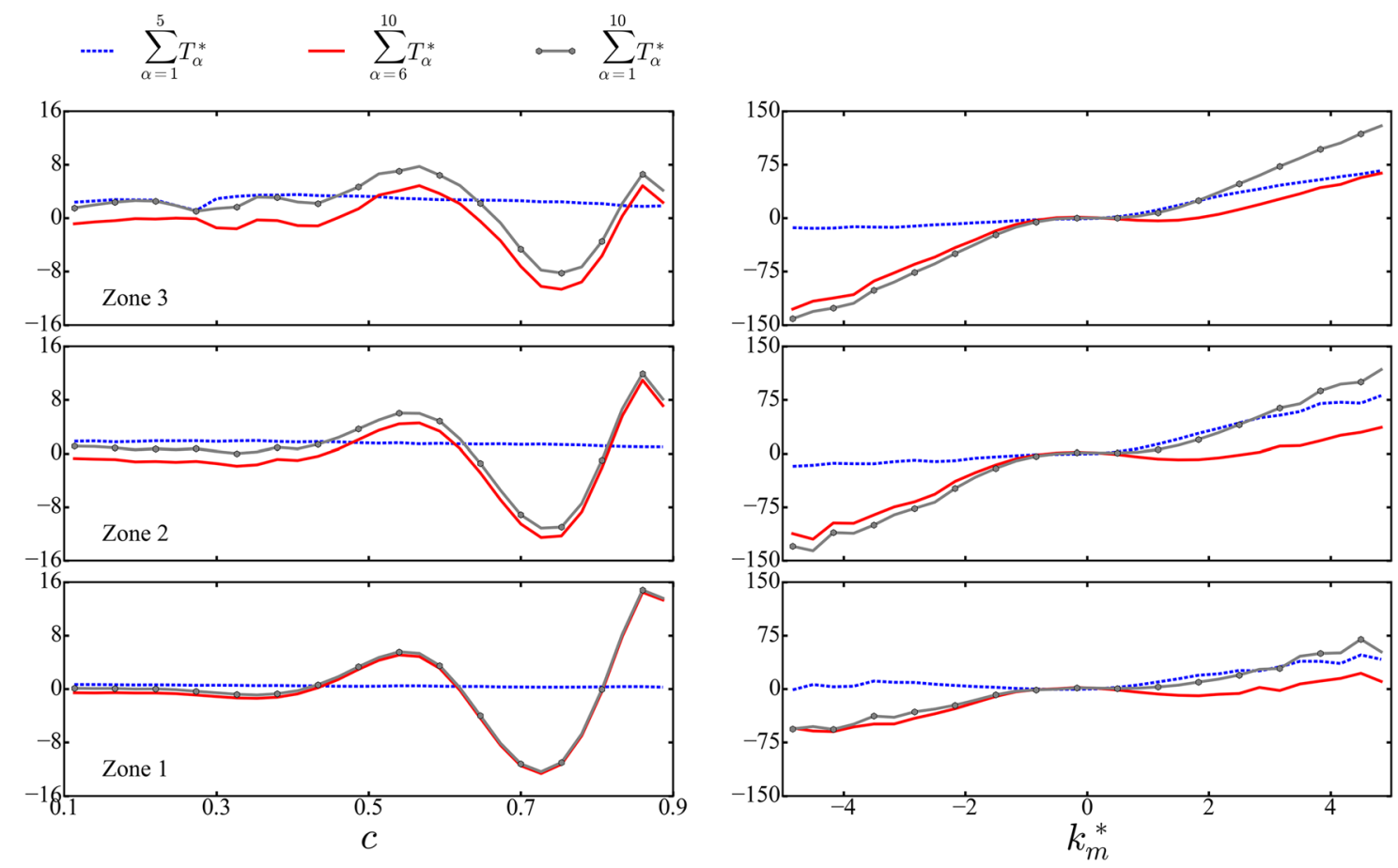

FIG. 5. Contribution of flow and added source terms and sum of all the terms, conditioned on the reaction progress variable $c$ and the mean curvature $k_{m}^{*}$. Results are separately examined in three zones (Zone 1: 5-20 mm, Zone 2: 20-45 mm, and Zone 3: 45-65 mm are shown in 3rd, 2nd, and 1st rows, respectively). 

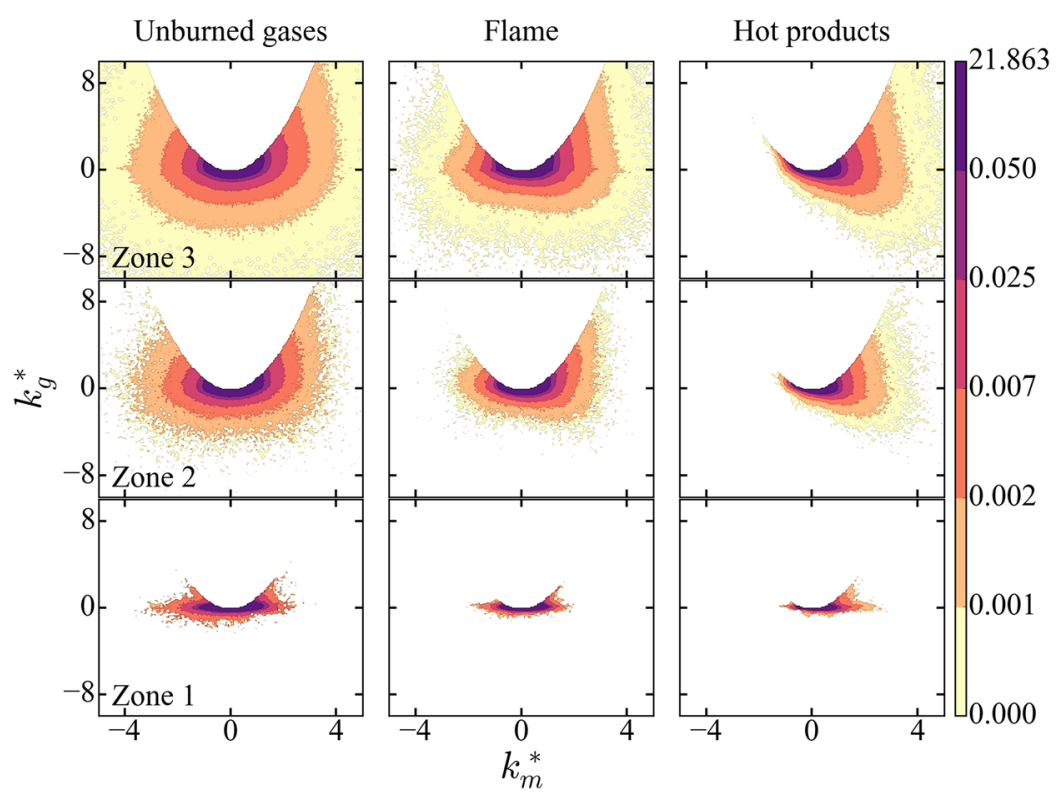

FIG. 6. JPDFs of the mean curvature $k_{m}^{*}$ and Gauss curvature $k_{g}^{*}$. Results are separately examined in three zones (Zone 1: 5-20 mm, Zone 2: $20-45 \mathrm{~mm}$, and Zone 3: 45-65 mm are shown in 3rd, 2nd, and 1st rows, respectively) and in three regions, namely, unburned gases $(c=0-0.1)$, flame $(c=0.1-0.9)$, and hot products $(c=$ $0.9-0.99)$

\section{Terms of the curvature evolution equation conditional upon the local geometry of iso-scalar surfaces}

To analyze the small-scale structures and local topologies, the joint probability density functions (JPDFs) of the mean and Gauss curvatures $k_{m}^{*}$ and $k_{g}^{*}$ for three regions, namely, unburned gases ( $c=0-0.1$ ), flame ( $c=0.1-0.9$ ), and hot products $(c=0.9-0.99)$, are shown in Fig. 6. It can be seen from Fig. 6 that the ranges of $k_{m}^{*}$ and $k_{g}^{*}$ broaden from Zone 1 to Zone 3 as the flame becomes increasingly wrinkled in the downstream direction. ${ }^{37,57}$ The JPDFs are presented in the various zones above the burner exit in order to identify the effects of the flame-turbulence interaction in the downstream direction. The regions unburned and products do not show a significant chemical activity (as expected), whereas a large positive dilation rate is found in the flame region due to heat release. ${ }^{37,57}$ It can be seen that nearly flat iso-scalar surfaces are the most probable geometries in all zones. Flat structures are evident very close to the burner exit where the flame wrinkling is weak. In the unburned gases, the JPDF appears to be symmetrical about $k_{m}^{*}=0$ and this behavior does not change with the axial distance. In the flame region, the JPDF is slightly skewed toward positive values of $k_{m}^{*}$, reflecting the features of evolving small-scale structures due to turbulence in the zone of intense chemical activity. This behavior is prominent in Zone 3 , where flame wrinkling increases due to turbulence effects. Toward the products region, the probability of finding concave scalar structures decreases drastically and nearly flat or convex geometries are most probable; this behavior can be attributed to the focusing of heat by concave geometries. Convex isosurfaces with moderate $k_{m}^{*}$ values, particularly the convex elliptical cup geometries, survive in the products region.

Figure 7 shows the mean values of the flow contributions $T_{1-5}^{*}$ to the generation/annihilation of mean curvature conditional upon the mean $k_{m}^{*}$ and Gauss $k_{g}^{*}$ curvatures. The mean values of the added contributions $T_{6-10}^{*}$ conditional upon $k_{m}^{*}$ and $k_{g}^{*}$ are shown in Fig. 8. Moderate and large positive values of the different terms, especially the major contributors $T_{3}$ and $T_{8}$, correlate with $k_{m}^{*}>0$, with iso-scalar surface geometries of a convex-elliptic nature, whereas the negative values
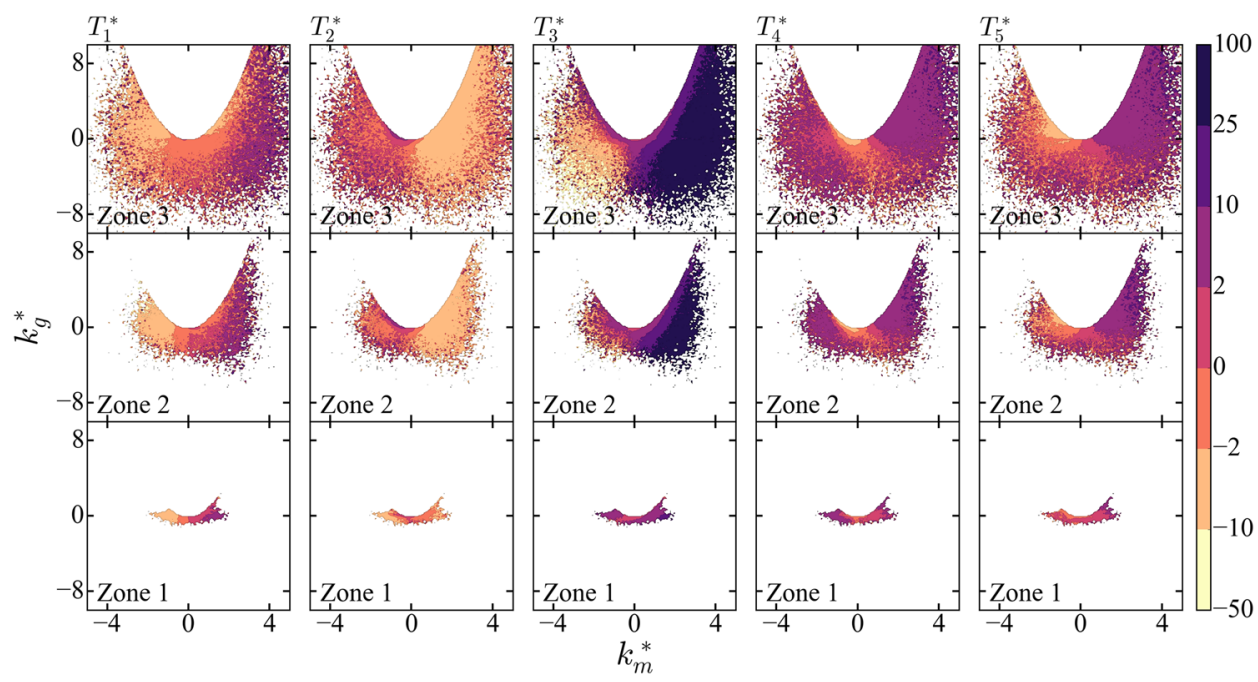

FIG. 7. Conditional mean of flow terms upon the mean curvature $k_{m}^{*}$ and Gauss curvature $k_{g}^{*}$ in the flame region $(c=0.1-$ $0.9)$. Results are separately examined in three zones (Zone 1: 5-20 mm, Zone 2: 20-45 mm, and Zone 3: 45-65 mm are shown in $3 \mathrm{rd}$, 2nd, and 1st rows, respectively). 


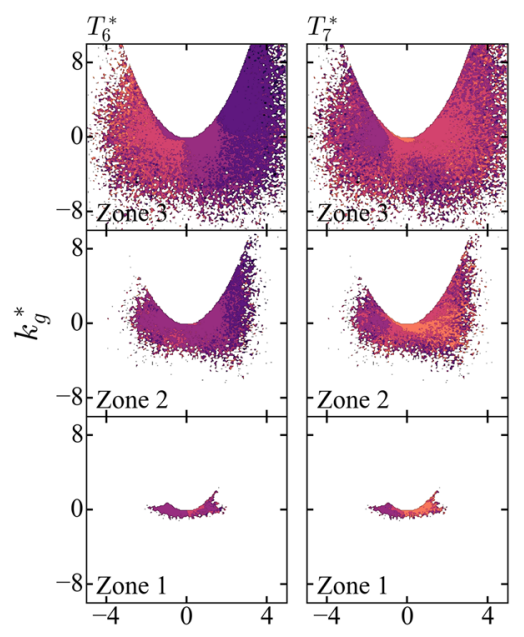

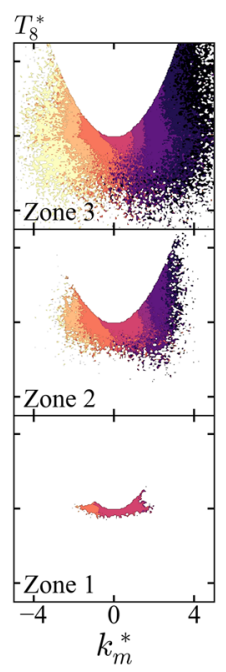

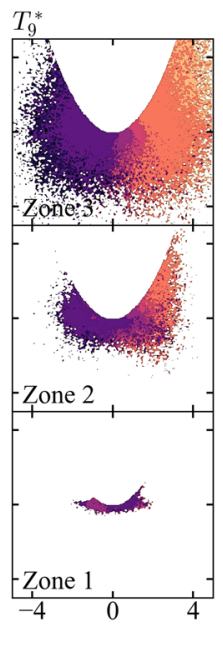

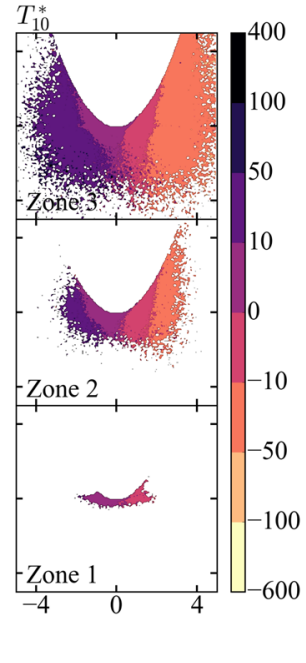

FIG. 8. Conditional mean of added terms upon the mean curvature $k_{m}^{*}$ and Gauss curvature $k_{g}^{*}$ in the flame region $(c=0.1-0.9)$. Results are separately examined in three zones (Zone 1: 5-20 mm, Zone 2: 20-45 $\mathrm{mm}$, and Zone 3: 45-65 $\mathrm{mm}$ are shown in 3rd, $2 \mathrm{nd}$, and 1 st rows, respectively). of these terms are associated with flat and concave geometries. These trends are in agreement with the observed results from Fig. 5 and also suggest that the negative (positive) values of the curvature transport equation correspond to concave (convex) scalar micro-structures. On the other hand, the main added contributions $T_{9}$ and $T_{10}$ display negative correlations with $k_{m}$ similar to those found in previous studies between the local volumetric dilatation rate and the mean flame curvature, ${ }^{17,78}$ where large positive dilatation rate values are obtained due to focusing of heat at concave geometries.

\section{E. Implications of the results}

The findings based on Figs. 3-5 and 7 reveal that the added contributions to the curvature $k_{m}$ arising from displacement speed $S_{d}$ play dominant roles in the reaction zone and the burned gas side of the flame front. This essentially indicates that curvature and displacement speed are intrinsically interlinked. Therefore, curvature not only affects the local displacement speed (a dominant negative correlation between $S_{d}$ and $k_{m}$ exists in this flame at all locations ${ }^{37,57}$ ), as this displacement governs the curvature evolution and thus flame wrinkling in turn. Moreover, the flow contribution arising from the flow stretching term $T_{3}=-2\left(S_{i j} n_{j, i}\right)$ and the contribution due to flow strain rate gradients $T_{4}=-\left(\partial S_{i j} / \partial x_{i}\right)\left(n_{j} / 2\right)$ are found to govern flame curvature evolution on the unburned gas side, where the reaction effects are not strong. As both the curvature and displacement speed have significant influences on the evolution of $|\nabla c|,{ }^{18,23,35-37}$ they have implications on FSD and SDR evolutions ${ }^{40-42}$ and thus on the closure of molecular mixing and micro-mixing in PDF methods. ${ }^{65,79}$ Thus, it is not surprising that the normal strain rate and displacement speed play key roles in the evolutions of curvature, SDF, FSD, and SDR. Furthermore, some of the behaviours of the instantaneous curvature transport equation will be visible for the resolved curvature in the context of LES so that the statistics of the displacement speed and flame normal strain rate (in other words, scalar gradient alignment with local principal strain rates) need to be adequately accounted for to accurately predict the resolved flame wrinkling. However, this necessity is not limited to the resolved scale, and the aforementioned physical mechanisms determine sub-grid scale wrinkling and influence the closure of sub-grid terms related to curvature in both the FSD and SDR transport equations. ${ }^{40-42}$

\section{CONCLUSIONS}

Flame resolved high-fidelity simulation data of a turbulent premixed bluff body burner have been used to examine the evolution of mean curvature. This involves the terms arising from the fluid flow and from the non-material nature of the propagating flame iso-surfaces. It has been shown that the contributions to the curvature evolution equation due to the fluid flow play an important role on the unburned gas side, whereas the added terms due to flame propagation dominate the reaction region and in the hot products. The curvature added flow stretching term $T_{8}=-2\left(S_{i j}^{a} n_{j, i}\right)$, which is a combination of the curvature tensor and gradients of flow and diffusion/chemically induced strain rates, is predominantly responsible for the generation/annihilation of curvature in the reaction zone and on the burnt side of the flame. On the contrary, the generation of mean curvature on the unburned side is governed by the flow stretching term $T_{3}=-2\left(S_{i j} n_{j, i}\right)$ and curvature generation by flow strain gradients $T_{4}=-\left(\partial S_{i j} / \partial x_{i}\right)\left(n_{j} / 2\right)$. The mean values of $T_{3}$ and $T_{8}$ conditioned upon the mean curvature $k_{m}$ and Gauss curvature $k_{g}$ indicate that the annihilation of flame curvature is prevalent at concave scalar iso-surfaces, whereas the generation of flame curvature occurs at convex scalar iso-surfaces. On the other hand, the added terms $T_{9}$ and $T_{10}$ are the main contributions to the flame curvature for concave scalar iso-surfaces. The present study also reveals that the mean curvature within the flame and especially in the reaction zone is controlled by the balance between micro-mixing and combustion, and future development of mixing models should incorporate some representation of mean curvature evolution to relate the coupling between small-scale flow strain rate, molecular diffusion, and chemical conversion.

\section{ACKNOWLEDGMENTS}

The authors are grateful to Jülich Supercomputing Centre (JSC), support by the state of NRW, EPSRC, and ARCHER for financial and computational support. This project has 
received funding from the European Union's Horizon 2020 research and innovation program under Grant Agreement No. 706672-ITPF.

${ }^{1}$ P. Pelcé, Dynamics of Curved Fronts (Academic Press, Inc., 1988).

${ }^{2}$ G. H. Markstein, "Experimental and theoretical studies of flame-front stability," in Dynamics of Curved Fronts, edited by P. Pelcé (Academic Press, Inc., 1988), pp. 413-423.

${ }^{3}$ P. Clavin and G. Joulin, "Premixed flames in large scale and high intensity turbulent flow," J. Phys. Lett. 44, 1-12 (1983).

${ }^{4}$ D. W. Mikolaitis, "The interaction of flame curvature and stretch, part 1: The concave premixed flame," Combust. Flame 57, 25-31 (1984).

${ }^{5} \mathrm{D}$. W. Mikolaitis, "The interaction of flame curvature and stretch, part 2: The concave premixed flame," Combust. Flame 58, 23-29 (1984).

${ }^{6}$ D. Haworth and T. Poinsot, "Numerical simulations of Lewis number effects in turbulent premixed flames," J. Fluid Mech. 244, 405-436 (1992).

${ }^{7} \mathrm{C}$. Rutland and A. Trouvé, "Direct simulations of premixed turbulent flames with nonunity Lewis numbers," Combust. Flame 94, 41-57 (1993).

${ }^{8}$ T. Echekki and J. H. Chen, "Unsteady strain rate and curvature effects in turbulent premixed methane-air flames," Combust. Flame 106, 184-202 (1996).

${ }^{9}$ B. Renou, A. Boukhalfa, D. Puechberty, and M. Trinité, "Effects of stretch on the local structure of freely propagating premixed low-turbulent flames with various Lewis numbers," Symp. (Int.) Combust. 27, 841-847 (1998).

${ }^{10}$ N. Peters, P. Terhoeven, J. H. Chen, and T. Echekki, "Statistics of flame displacement speeds from computations of 2-D unsteady methane-air flames," Symp. (Int.) Combust. 27, 833-839 (1998).

${ }^{11}$ T. Echekki and J. H. Chen, "Analysis of the contribution of curvature to premixed flame propagation," Combust. Flame 118, 308-311 (1999).

${ }^{12}$ J. H. Chen and H. G. Im, "Correlation of flame speed with stretch in turbulent premixed methane/air flames," Symp. (Int.) Combust. 27, 819-826 (1998).

${ }^{13}$ J. B. Chen and H. G. Im, "Stretch effects on the burning velocity of turbulent premixed hydrogen/air flames," Proc. Combust. Inst. 28, 211-218 (2000).

${ }^{14}$ E. R. Hawkes and J. H. Chen, "Direct numerical simulation of hydrogenenriched lean premixed methane-air flames," Combust. Flame 138, 242258 (2004).

${ }^{15}$ E. R. Hawkes and J. H. Chen, "Evaluation of models for flame stretch due to curvature in the thin reaction zones regime," Proc. Combust. Inst. 30, 647-655 (2005).

${ }^{16} \mathrm{~N}$. Chakraborty and S. Cant, "Unsteady effects of strain rate and curvature on turbulent premixed flames in an inflow-outflow configuration," Combust. Flame 137, 129-147 (2004).

${ }^{17} \mathrm{~N}$. Chakraborty and R. Cant, "Influence of Lewis number on curvature effects in turbulent premixed flame propagation in the thin reaction zones regime," Phys. Fluids 17, 105105 (2005).

${ }^{18}$ N. Chakraborty and R. Cant, "Effects of strain rate and curvature on surface density function transport in turbulent premixed flames in the thin reaction zones regime," Phys. Fluids 17, 065108 (2005).

${ }^{19}$ K. Jenkins, M. Klein, N. Chakraborty, and R. Cant, "Effects of strain rate and curvature on the propagation of a spherical flame kernel in the thinreaction-zones regime," Combust. Flame 145, 415-434 (2006).

${ }^{20} \mathrm{M}$. Klein, N. Chakraborty, K. Jenkins, and R. Cant, "Effects of initial radius on the propagation of premixed flame kernels in a turbulent environment," Phys. Fluids 18, 055102 (2006).

${ }^{21}$ V. R. Savarianandam and C. Lawn, "Burning velocity of premixed turbulent flames in the weakly wrinkled regime," Combust. Flame 146, 1-18 (2006).

${ }^{22} \mathrm{~N}$. Chakraborty, "Comparison of displacement speed statistics of turbulent premixed flames in the regimes representing combustion in corrugated flamelets and thin reaction zones," Phys. Fluids 19, 105109 (2007).

${ }^{23} \mathrm{~N}$. Chakraborty, E. Hawkes, J. Chen, and R. Cant, "The effects of strain rate and curvature on surface density function transport in turbulent premixed methane-air and hydrogen-air flames: A comparative study," Combust. Flame 154, 259-280 (2008).

${ }^{24}$ I. Han and K. Y. Huh, "Roles of displacement speed on evolution of flame surface density for different turbulent intensities and Lewis numbers in turbulent premixed combustion," Combust. Flame 152, 194-205 (2008).

${ }^{25}$ G. Hartung, J. Hult, R. Balachandran, M. Mackley, and C. Kaminski, "Flame front tracking in turbulent lean premixed flames using stereo PIV and timesequenced planar LIF of OH,” Appl. Phys. B 96, 843-862 (2009).

${ }^{26}$ N. Chakraborty, M. Klein, and R. Cant, "Effects of turbulent Reynolds number on the displacement speed statistics in the thin reaction zones regime of turbulent premixed combustion,” J. Combust. 2011, 473679.
${ }^{27}$ N. Chakraborty, G. Hartung, M. Katragadda, and C. Kaminski, "Comparison of 2D and 3D density-weighted displacement speed statistics and implications for laser based measurements of flame displacement speed using direct numerical simulation data," Combust. Flame 158, 1372-1390 (2011).

${ }^{28}$ J. Kerl, C. Lawn, and F. Beyrau, "Three-dimensional flame displacement speed and flame front curvature measurements using quad-plane PIV," Combust. Flame 160, 2757-2769 (2013).

${ }^{29}$ G. K. Giannakopoulos, M. Matalon, C. Frouzakis, and A. G. Tomboulides, "The curvature Markstein length and the definition of flame displacement speed for stationary spherical flames," Proc. Combust. Inst. 35, 737-743 (2015).

${ }^{30}$ A. Marshall, J. Lundrigan, P. Venkateswaran, J. Seitzman, and T. Lieuwen, "Fuel effects on leading point curvature statistics of high hydrogen content fuels," Proc. Combust. Inst. 35, 1417-1424 (2015).

${ }^{31}$ A. Aspden, J. Bell, M. Day, and F. Egolfopoulos, "Turbulence-flame interactions in lean premixed dodecane flames," Proc. Combust. Inst. 36, 2005-2016 (2017).

${ }^{32}$ S. Pope, "The evolution of surfaces in turbulence," Int. J. Eng. Sci. 26, 445-469 (1988)

${ }^{33}$ S. M. Candel and T. J. Poinsot, "Flame stretch and the balance equation for the flame area," Combust. Sci. Technol. 70, 1-15 (1990).

${ }^{34}$ L. Vervisch, E. Bidaux, K. Bray, and W. Kollmann, "Surface density function in premixed turbulent combustion modeling, similarities between probability density function and flame surface approaches," Phys. Fluids 7, 2496-2503 (1995).

${ }^{35} \mathrm{~N}$. Chakraborty and M. Klein, "Influence of Lewis number on the surface density function transport in the thin reaction zone regime for turbulent premixed flames," Phys. Fluids 20, 065102 (2008).

${ }^{36} \mathrm{~N}$. Chakraborty and M. Klein, "Effects of global flame curvature on surface density function transport in turbulent premixed flame kernels in the thin reaction zones regime," Proc. Combust. Inst. 32, 1435-1443 (2009).

${ }^{37}$ A. Sandeep, F. Proch, A. M. Kempf, and N. Chakraborty, "Statistics of strain rates and surface density function in a flame-resolved high-fidelity simulation of a turbulent premixed bluff body burner," Phys. Fluids 30, 065101 (2018).

${ }^{38}$ S. Girimaji and S. Pope, "Propagating surfaces in isotropic turbulence," J. Fluid Mech. 234, 247-277 (1992).

${ }^{39}$ Y. Gao, N. Chakraborty, and N. Swaminathan, "Local strain rate and curvature dependences of scalar dissipation rate transport in turbulent premixed flames: A direct numerical simulation analysis," J. Combust. 2014, 280671.

${ }^{40}$ N. Chakraborty and R. Cant, "A priori analysis of the curvature and propagation terms of the flame surface density transport equation for large eddy simulation," Phys. Fluids 19, 105101 (2007).

${ }^{41} \mathrm{~N}$. Chakraborty and R. Cant, "Direct numerical simulation analysis of the flame surface density transport equation in the context of large eddy simulation," Proc. Combust. Inst. 32, 1445-1453 (2009).

${ }^{42}$ Y. Gao, N. Chakraborty, and N. Swaminathan, "Scalar dissipation rate transport and its modeling for large eddy simulations of turbulent premixed combustion," Combust. Sci. Technol. 187, 362-383 (2015).

${ }^{43}$ J. Kwon, Y. Park, and K. Y. Huh, "Flamelet characteristics at the leading edge and through the flame brush of statistically steady incompressible turbulent premixed flames," Combust. Flame 164, 85-98 (2016).

${ }^{44}$ M. Klein, N. Chakraborty, and R. Cant, "Effects of turbulence on selfsustained combustion in premixed flame kernels: A direct numerical simulation (DNS) study," Flow, Turbul. Combust. 81, 583-607 (2008).

${ }^{45} \mathrm{H}$. A. Uranakara, S. Chaudhuri, and K. Lakshmisha, "On the extinction of igniting kernels in near-isotropic turbulence," Proc. Combust. Inst. 36, 1793-1800 (2017).

${ }^{46} \mathrm{P}$. Clavin and F. Williams, "Effect of Lewis number on propagation of wrinkled flames in turbulent flow," in Combustion in Reactive Systems, Progress in Astronautics and Aeronautics Vol. 76, edited by J. R. Bowen, R. I. Soloukhin, N. Manson, and A. K. Oppenheim (AIAA, 1981), pp. 403-411.

${ }^{47}$ G. I. Sivashinsky, "Instabilities, pattern formation, and turbulence in flames," Annu. Rev. Fluid Mech. 15, 179-199 (1983).

${ }^{48}$ F. Creta, R. Lamioni, P. E. Lapenna, and G. Troiani, "Interplay of DarrieusLandau instability and weak turbulence in premixed flame propagation," Phys. Rev. E 94, 053102 (2016).

${ }^{49}$ I. Shepherd and W. T. Ashurst, "Flame front geometry in premixed turbulent flames," Symp. (Int.) Combust. 24, 485-491 (1992).

${ }^{50} \mathrm{~W}$. T. Ashurst and I. Shepherd, "Flame front curvature distributions in a turbulent premixed flame zone," Combust. Sci. Technol. 124, 115-144 (1997). 
${ }^{51}$ T.-W. Lee, G. North, and D. Santavicca, "Curvature and orientation statistics of turbulent premixed flame fronts," Combust. Sci. Technol. 84, 121-132 (1992).

${ }^{52}$ T.-W. Lee, G. North, and D. Santavicca, "Surface properties of turbulent premixed propane/air flames at various Lewis numbers," Combust. Flame 93, 445-456 (1993).

${ }^{53}$ S. Gashi, J. Hult, K. W. Jenkins, N. Chakraborty, S. Cant, and C. F. Kaminski, "Curvature and wrinkling of premixed flame kernels-Comparisons of $\mathrm{OH}$ PLIF and DNS data," Proc. Combust. Inst. 30, 809-817 (2005).

${ }^{54}$ F. T. Yuen and Ö. L. Gülder, "Premixed turbulent flame front structure investigation by Rayleigh scattering in the thin reaction zone regime," Proc. Combust. Inst. 32, 1747-1754 (2009).

${ }^{55}$ H. Wang, E. R. Hawkes, B. Zhou, J. H. Chen, Z. Li, and M. Aldén, "A comparison between direct numerical simulation and experiment of the turbulent burning velocity-related statistics in a turbulent methane-air premixed jet flame at high Karlovitz number," Proc. Combust. Inst. 36, 2045-2053 (2017).

${ }^{56}$ C. Dopazo, J. Martin, L. Cifuentes, and J. Hierro, "Strain, rotation and curvature of non-material propagating iso-scalar surfaces in homogeneous turbulence," Flow, Turbul. Combust. 101, 1-32 (2018).

${ }^{57}$ F. Proch, P. Domingo, L. Vervisch, and A. M. Kempf, "Flame resolved simulation of a turbulent premixed bluff-body burner experiment. Part I: Analysis of the reaction zone dynamics with tabulated chemistry," Combust. Flame 180, 321-339 (2017).

${ }^{58}$ M. S. Sweeney, S. Hochgreb, M. J. Dunn, and R. S. Barlow, "The structure of turbulent stratified and premixed methane/air flames I: Non-swirling flows," Combust. Flame 159, 2896-2911 (2012).

${ }^{59}$ M. S. Sweeney, S. Hochgreb, M. J. Dunn, and R. S. Barlow, "The structure of turbulent stratified and premixed methane/air flames II: Swirling flows," Combust. Flame 159, 2912-2929 (2012).

${ }^{60}$ R. Zhou, S. Balusamy, M. S. Sweeney, R. S. Barlow, and S. Hochgreb, "Flow field measurements of a series of turbulent premixed and stratified methane/air flames," Combust. Flame 160, 2017-2028 (2013).

${ }^{61}$ M. Euler, R. Zhou, S. Hochgreb, and A. Dreizler, "Temperature measurements of the bluff body surface of a swirl burner using phosphor thermometry," Combust. Flame 161, 2842-2848 (2014).

${ }^{62}$ M. M. Kamal, R. Zhou, S. Balusamy, and S. Hochgreb, "Favre-and Reynolds-averaged velocity measurements: Interpreting PIV and LDA measurements in combustion," Proc. Combust. Inst. 35, 3803-3811 (2015).

${ }^{63}$ M. M. Kamal, R. S. Barlow, and S. Hochgreb, "Conditional analysis of turbulent premixed and stratified flames on local equivalence ratio and progress of reaction," Combust. Flame 162, 3896-3913 (2015).

${ }^{64}$ C. Dopazo, L. Cifuentes, J. Martin, and C. Jimenez, "Strain rates normal to approaching iso-scalar surfaces in a turbulent premixed flame," Combust. Flame 162, 1729-1736 (2015).
${ }^{65}$ C. Dopazo, L. Cifuentes, J. Hierro, and J. Martin, "Micro-scale mixing in turbulent constant density reacting flows and premixed combustion," Flow, Turbul. Combust. 96, 547-571 (2016).

${ }^{66} \mathrm{C}$. Dopazo and L. Cifuentes, "The physics of scalar gradients in turbulent premixed combustion and its relevance to modeling," Combust. Sci. Technol. 188, 1376-1397 (2016).

${ }^{67}$ C. Dopazo, J. Martin, and J. Hierro, "Local geometry of iso-scalar surfaces," Phys. Rev. E 76, 056316 (2007).

${ }^{68}$ R. Mercier, B. Fiorina, F. Proch, and A. M. Kempf, "Numerical and modeling strategies for the simulation of the Cambridge stratified flame series," TSFP Digital Library Online, 2013.

${ }^{69}$ R. Mercier, T. Schmitt, D. Veynante, and B. Fiorina, "The influence of combustion SGS submodels on the resolved flame propagation. Application to the LES of the Cambridge stratified flames," Proc. Combust. Inst. 35, 1259-1267 (2015).

${ }^{70}$ F. Proch and A. M. Kempf, "Numerical analysis of the Cambridge stratified flame series using artificial thickened flame LES with tabulated premixed flame chemistry," Combust. Flame 161, 2627-2646 (2014).

${ }^{71}$ B. Fiorina, O. Gicquel, L. Vervisch, S. Carpentier, and N. Darabiha, "Approximating the chemical structure of partially premixed and diffusion counterflow flames using FPI flamelet tabulation," Combust. Flame 140, 147-160 (2005).

${ }^{72}$ P.-D. Nguyen, L. Vervisch, V. Subramanian, and P. Domingo, "Multidimensional flamelet-generated manifolds for partially premixed combustion," Combust. Flame 157, 43-61 (2010).

${ }^{73}$ J. van Oijen and L. de Goey, "Modelling of premixed laminar flames using flamelet-generated manifolds," Combust. Sci. Technol. 161, 113-137 (2000).

${ }^{74}$ N. Peters, Turbulent Combustion (Cambridge University Press, 2000).

${ }^{75} \mathrm{M}$. Boger, D. Veynante, H. Boughanem, and A. Trouvé, "Direct numerical simulation analysis of flame surface density concept for large eddy simulation of turbulent premixed combustion," Symp. (Int.) Combust. 27, 917-925 (1998).

${ }^{76}$ N. Chakraborty and N. Swaminathan, "Influence of the Damköhler number on turbulence-scalar interaction in premixed flames. I. Physical insight," Phys. Fluids 19, 045103 (2007).

${ }^{77}$ G. Hartung, J. Hult, C. Kaminski, J. Rogerson, and N. Swaminathan, "Effect of heat release on turbulence and scalar-turbulence interaction in premixed combustion," Phys. Fluids 20, 035110 (2008).

${ }^{78}$ L. Cifuentes, C. Dopazo, J. Martin, P. Domingo, and L. Vervisch, "Recent developments in PDF methods," Proc. Combust. Inst. 35, 1295-1303 (2015).

${ }^{79} \mathrm{C}$. Dopazo, "Local volumetric dilatation rate and scalar geometries in a premixed methane-air turbulent jet flame," in Turbulent Reacting Flows (Academic Press, London, 1994), pp. 375-474. 


\section{DuEPublico}

Duisburg-Essen Publications online

\section{DES SE B B R R G}

Offen im Denken

Ub $\mid \begin{aligned} & \text { universitäts } \\ & \text { bibliothek }\end{aligned}$

This text is made available via DuEPublico, the institutional repository of the University of Duisburg-Essen. This version may eventually differ from another version distributed by a commercial publisher.

DOI: $\quad 10.1063 / 1.5044525$

URN： urn:nbn:de:hbz:464-20191209-102506-1

(C) 2018 Author(s). This article may be downloaded for personal use only. Any other use requires prior permission of the authors and AIP Publishing.

All rights reserved. 